\title{
Numerical Analysis on Aerodynamic Performance of Counter-rotating Wind Turbine through Rear Rotor Configuration
}

\author{
Verdy A. Koehuan ${ }^{1,2}$, Sugiyono ${ }^{1} \&$ Samsul Kamal ${ }^{1}$ \\ ${ }^{1}$ Department of Mechanical and Industrial Engineering, Faculty of Engineering, Gadjah Mada University, \\ Yogyakarta 55281, Indonesia \\ ${ }^{2}$ Department of Mechanical Engineering, Faculty of Science and Engineering, University of Nusa Cendana, \\ Kupang 85001, Indonesia \\ Correspondence: Verdy A. Koehuan, Department of Mechanical Engineering, Faculty of Science and Engineering, \\ University of Nusa Cendana, Kupang 85001, Indonesia. Tel: 62-852-2022-2804. E-mail: \\ verdy.koehuan@staf.undana.ac.id
}

Received: Nov. 23, 2018

Accepted: Dec. 4, $2018 \quad$ Online Published: January 26, 2019

doi:10.5539/mas.v13n2p240

URL: https://doi.org/10.5539/mas.v13n2p240

\begin{abstract}
Numerical analysis was conducted on the aerodynamic performance and the flow characteristics around the counter-rotating wind turbine or CRWT blade through rear rotor configuration using various rotor diameter ratios and distance ratios to the turbine blade through a CFD (Computational Fluid Dynamics) simulation. CFD simulation showed the normalized power coefficients of the front rotor, rear rotor, and combined rotor (CRWT) to the single rotor with a strong influence of the rear rotor configuration with the addition of tip speed ratio (TSR). A larger average normalized power coefficient takes place at $\mathrm{D}_{1} / \mathrm{D}_{2}=1.0$ with $\mathrm{L} / \mathrm{D}_{1}=0.75$ by 1.221 . It is about $22.1 \%$ increased to the SRWT for the given TSR range. Axial velocity contours and resultant velocity vectors around the CRWT blade with a diameter ratio of $\mathrm{D}_{1} / \mathrm{D}_{2}>1.0$ and a closer rotor distance provide a stronger bound vortex and strong separation around the rear hub blade with a tendency to increase from the hub to the tip blade at low TSR. The higher the TSR, the movement of tip vortex moves closer to the rear tip blade which has the effect of increasing the leakage flow in the area of $\mathrm{D}_{1} / \mathrm{D}_{2}<1.0$.
\end{abstract}

Keywords: wind turbine, counter-rotating, power coefficient, double rotor, rear rotor configuration, computational fluid dynamics simulation

\section{Introduction}

The development of horizontal axis wind turbine (HAWT) three blades propeller technology today shows a very rapid progress, marked by an increase in turbine efficiency and a decrease in cost per kWh. Researches on the models and geometry shapes of turbine rotor blades with new airfoil profiles are very intense to improve aerodynamic efficiency in order to maximize the power which can be generated by turbines from wind energy content in free air flow. The wind turbine rotor is the main key in the process of changing wind kinetic energy into mechanical energy. Therefore, the aerodynamic blade geometry design with optimum efficiency is absolutely needed to maximize the convertible energy.

Horizontal axis wind turbine three blades type propeller with a double rotor is known as double rotor wind turbine (DRWT) which has two rotors placed on one axis. DRWT with counter-rotating direction which is better known as counter-rotating wind turbine (CRWT) can generate electricity with or without using a transmission system. This wind turbine has two rotors of the same or different diameter, equally moving one alternator with the direction of rotation opposite each other. Aerodynamically, it can increase the power coefficient of two rotor wind turbines higher than single rotor condition. Large rotors are placed in front of a small rotor or vice versa. The rotational speed of these two rotors is quite synchronous working together and supporting each other in driving the alternator (Kanemoto and Galal, 2006).

Numerical and experimental aerodynamic studies have been carried out by several researchers showing that the efficiency of dual-rotor wind turbines is better than a single rotor. The development of CRWT continues to be carried out through numerical and experimental modeling to maximize the wind power and minimize the energy costs per kWh of a generating system. Ushiyama et al. (1996) conducted tests on dual rotor wind turbines with the 
principle of counter-rotating and co-axial through variations in the number of blades with a non-dimensional rotor diameter of $D_{1} / D_{2}=0.5$, where $D_{1}$ is the front rotor diameter and $D_{2}$ is the rear rotor diameter. It has to report an increase in the turbine power coefficient with a low starting torque on the counter-rotating turbine. This study shows that the counter-rotating wind turbine is more efficient than co-axial and can technically be used as an alternator drive for electricity generation because the relative rotation is quite high. Appa (2002) through his experiment on a $6 \mathrm{~kW}$ double rotating wind turbine prototype with the same diameter and two blade counts, shows that the addition of the rear rotor resulted in an additional of $30 \%$ output power. Habash et al. (2011) conducted experiment on small-scale contra-rotating wind turbines to determine the performance of the turbine with the same rotor diameter and the non-dimensional distance $\mathrm{L} / \mathrm{D}_{1}$, where $\mathrm{L}$ is the distance between the two rotors varied from 0.3 to 2.34 indicating that the counter-rotational system can convert wind energy up to $60 \%$. Tian et al. (2013) studied the effect of rotor rotation through experiments scale with the same rotor diameter doing rotor distance variations from 0.7 to 7.8 , obtained at a distance ratio of 0.7 with a counter-rotating $2 \mathrm{MW}$ wind turbine in atmospheric conditions. The boundary layer (atmospheric boundary layer) has a maximum power increase of $10 \%$ counter-rotating higher than co-axial. Ozbay et al. (2016) studied in more detail on the wake flow around the 2MW turbine rotor through experiments scale with the PIV (particle image velocimetry) method on the same rotor diameter as the rotor distance ratio of 0.25 . The counter-rotating turbine maximum power increases by $60 \%$ which is higher than a single rotor.

The aerodynamic study of the counter-rotating wind turbine rotor (CRWT) shows the best performance with a number of three blades, both in the front and rear rotors, while the co-axial wind turbine (same rotation direction) is the best performance with the number of two blades for each rotor. Experimental studies conducted by Merchant et al. (2009) which tested several blade models designed for counter-rotating showed a maximum power increase of $101.4 \%$ at $25 \mathrm{mph}$. Yuan et al. (2014) and Ozbay et al. (2014) also showed the same result by studying the effect of rotor rotation direction through experiments scale with the same rotor diameter on $2 \mathrm{MW}$ counter-rotating wind turbines. Mitulet et al. (2015) also experimentally applied a single rotor airfoil on counter-rotating which can improve CRWT performance.

Analytical solutions performed by Jung et al. (2005); Shen et al. (2007); Lee et al. (2010; 2012; 2013); and Hwang et al. (2013) by using a combination of blade element momentum and vortex methods showing an increase in the performance of a double rotor turbine with a single rotor. Lee et al. $(2010 ; 2012 ; 2013)$ despite successfully analyzing aerodynamic characteristics and interference in the wind turbine rotor counter-rotating through numerical solutions. In this analysis, however, the flow is not taken into account with certain turbulence levels which have an impact on the lack of accuracy in predicting vortex structure due to CRWT rotor rotation. The results of this study cannot be predicted precisely the performance of CRWT with the configuration of the diameter ratio and the distance ratio due to the lack of accurate prediction of rotor dynamic forces caused by vortices in turbulent flow. This is because the assumption is that the analysis of non-viscous flow so that the flow characteristics due to turbulence effects are not taken into account in the analysis of turbine rotor performance. However, the results of these studies indicate that the double rotor wind turbines are better performing if the direction of the front rotor rotation and rear rotor are counter-rotating.

The wake effect on the aerodynamic performance of CRWT consists of two parts, namely the near wake and far wake regions (Sorensen \& Shen, 2002). Near wake confirms the growth of flow wake characterized by a decrease in flow velocity, vortex, and turbulent kinetic energy around the front rotors and rear rotors of CRWT (Koehuan et al., 2017b). Experimental studies of near wake on a laboratory scale of CRWT were done by Tian et al. (2013), Yuan et al. (2014), Ozbay et al. (2014a), Ozbay et al. (2014b), Wang et al. (2016), and Ozbay et al. (2016). These studies were only carried out partially on the diameter ratio and the ratio of certain rotor axial distances from CRWT with limited operating conditions on the tip speed ratio. Therefore, the performance of CRWT on near wake of flow field and vortex structure cannot be explained in a wider tip speed ratio condition.

Rosenberg et al. (2014) and Moghadassian et al. (2016) successfully predicted that the performance and flow phenomena in double rotor wind turbines or DRWT with turbulent level conditions in the inflow are uniforms, stable, and neutral. However, it is not discussed in more detail about the interference wake at the near wake, so that the flow characteristic cannot be described for changes in the diameter ratio and axial distance of the two rotors and the CRWT operating conditions in the wider TSR range.

Various types of the blade (airfoil) have been used for the design and analysis of counter-rotating wind turbines, where each study is more likely to be carried out partially related to certain parameters such as diameter ratio, distance ratio, and tip speed ratio. Investigation of the effect of a blade model as input parameters on power coefficient $\left(\mathrm{C}_{\mathrm{P}}\right)$ counter-rotating wind turbines with variations in rotor diameter ratios and rotor distance ratios was carried out on certain tip speed ratios (TSR) or in relatively narrow ranges. Therefore, a comprehensive study that 
uses one type of blade by varying the various parameters existed in a wide enough range on the aerodynamic performance of counter-rotating wind turbine through rear rotor configurations and flow field phenomena with a certain turbulence level. Investigations carried out on the ratio of rotor diameter and distance ratio against TSR that is wide enough can show better turbine performance with wider operating conditions on the TSR.

This study aims to perform numerical analysis on aerodynamic performance and flow field phenomenon around the CRWT blade or near wake of counter-rotating wind turbine through various rear rotor configurations. Aerodynamic studies are carried out on horizontal axis wind turbine rotors with counter-rotating using the S826 airfoil for blade model as input parameters. The input parameters using rear rotor configurations as follows: diameter ratios of $\mathrm{D}_{1} / \mathrm{D}_{2}$ by $0.5,0.75,1.0,1.25$, and $1.5\left(\mathrm{D}_{1}\right.$ and $\mathrm{D}_{2}$ are front and rear rotor diameter) and rotor distance ratios of $\mathrm{L} / \mathrm{D}_{1}$ by 0.2 to 1.5 was carried out with wider operating conditions of tip speed ratio (TSR=1 to $\mathrm{TSR}=10$ ). The front rotor used the same diameter and operating condition with the single rotor wind turbine.

\section{Numerical Method}

\subsection{Numerical Models and Turbulent Models}

The numerical approach by CFD (computational fluid dynamics) simulation successfully analyzed 3D (threedimensional) flow phenomena at CRWT through the assumption of certain turbulence intensities in CRWT rotor inflow. Kumar et al. $(2012,2013)$ used the standard $k-e$ turbulence model and the standard $k-\omega$ shear stress transport turbulence model in a cylindrical domain of CRWT using tetrahedral mesh have good agreement with experiment. Hoang \& Yang (2013) used shear stress transport (SST) turbulence model in the periodicity of the $120^{\circ}$ computational domain of CRWT using hex mesh. Rosenberg et al. (2014) and Moghadassian et al. (2016) successfully predicted the performance and flow phenomena in double rotor wind turbines or DRWT using large eddy simulations (LES) for uniform inflow with no incoming turbulence and two atmospheric stability conditions: neutral and stable. Vasel-Be-Hagh \& Archer (2017) used large eddy simulations for analyzed the wind farms with counter-rotating wind turbines that it was found to produce $22.6 \%$ more power than the conventional wind farm.

The turbulent model of Reynolds-averaged Navier-Stokes equation for 3-D, incompressible, and steady flow condition uses $\mathrm{k}-\varepsilon$ model of realizable that is a semi-empirical model based on the transport equation of flow. Modeling of CRWT using $k-\varepsilon$ model of realizable is still low cost by economic in commercial used and low timeconsuming in terms of computational capacity and effort as reported by Taha et al. (2010), Hallanger \& Sand (2013), and Koehuan et al. (2017a). Model for turbulent kinetic energy (k) and its level of dissipation ( $(\varepsilon)$ which decreases the $k-\varepsilon$ model, it is assumed that the fully turbulent flow. One of the problems in turbulent modeling is the lack of accurate prediction of flow separation on the surface with adverse pressure gradient conditions which is very important in the aerodynamics of wind turbine rotors. However, complex flow problems such as separation and pressure gradients will be better prediction through turbulent $k-\varepsilon$ models of realizable with wall treatment approaches such as non-equilibrium wall functions.

The numerical solution procedure with ANSYS Fluent converts the Navier-Stokes non-linear differential equations into a set of algebraic equations using a pressure-based solver. Mesh geometry is the key to the level of accuracy and the length of the calculation process because of the number of cells, the better the numerical results, but of course the longer the time. The type of solver used is pressure-based with absolute speed formulations, while the solution scheme for pressure-velocity coupling uses coupled algorithm. In the iteration procedure, the solution method for solving pressure equations uses second order, while for momentum and turbulence equations uses QUICK, which corresponds to the type of mesh used that is hexahedral so that the accuracy of the calculation will be better. The simulation process in this study is run in parallel using Fluent Ansys 17.0 through a computer unit with specifications: Intel Core i3 4130CPU processor, 16GB memory, VGA NVidia GeForce GT 730 2GB.

The aerodynamic performance of dual-rotor wind turbines counter-rotating against variations in input parameters that are numerically studied on aerodynamic performance and flow field phenomena around the CRWT blade (near wake) with a certain turbulence intensity. CFD simulations are performed on SRWT and CRWT rotor blades with a third computational domain through the assumption of symmetrical flow by applying periodic interfaces to their boundary conditions. The solidity and angle of the first and second rotor blades are made constant, while the first rotor and second rotor TSR (tip speed ratio) are obtained from the first and second rotor rotation ratios of $\mathrm{n}_{2} / \mathrm{n}_{1}=0.6$.

Turbulent kinetic energy $k$ and the dissipation rate of $\varepsilon$ obtained from the general equation of fluid flow are the Reynolds-averaged Navier-Stokes (RANS) equations (Fluent Inc, 2006).

$$
\frac{\partial}{\partial t}(\rho k)+\frac{\partial}{\partial x_{i}}\left(\rho k u_{i}\right)=\frac{\partial}{\partial x_{j}}\left[\left(\mu+\frac{\mu_{t}}{\sigma_{k}}\right) \frac{\partial k}{\partial x_{j}}\right]+G_{k}+G_{b}-\rho \varepsilon-Y_{M}+S_{k}
$$




$$
\frac{\partial}{\partial t}(\rho \varepsilon)+\frac{\partial}{\partial x_{i}}\left(\rho \varepsilon u_{i}\right)=\frac{\partial}{\partial x_{j}}\left[\left(\mu+\frac{\mu_{t}}{\sigma_{\varepsilon}}\right) \frac{\partial \varepsilon}{\partial x_{j}}\right]+C_{1 \varepsilon} \frac{\varepsilon}{k}\left(G_{k}+C_{3 \varepsilon} G_{b}\right)-C_{2 \varepsilon} \rho \frac{\varepsilon^{2}}{k}+S_{\varepsilon}
$$

Where $-\rho \overline{u_{i}^{\prime} u_{j}^{\prime}}$ Reynolds stresses with components of speed $u_{i}=\bar{u}_{i}+u_{i}^{\prime} . G_{k}$ is the generation of turbulent kinetic energy due to the average velocity gradient, $G_{b}$ of turbulent kinetic generation due to buoyancy, $Y_{M}$ represents contribution due to compressibility and $k=\overline{0.5 u_{i}^{\prime} u_{j}^{\prime}}$ mean turbulent kinetic energy. $C_{1 \varepsilon}, C_{2 \varepsilon}$, and $C_{3 \varepsilon}$ are constants; $\sigma_{k}$ and $\sigma_{\varepsilon}$ are Prandtl turbulent numbers for $k$ and $\varepsilon$. The turbulent viscosity $\mu_{t}$ calculated from combining $k$ and $\varepsilon$ with $\mu_{t}=\rho C_{\mu} k^{2} / \varepsilon$, where $C_{\mu}$ is a constant. The constants used in the formula above taken based on the default.

\subsection{Blade Geometry and Boundary Conditions}

The blade model used in this study is a three blades type horizontal axis wind turbine adopted from the blade model developed at the Norwegian Center for Offshore Wind Energy and the Department of Energy and Process Engineering, Norwegian University of Science and Technology NTNU, Trondheim, Norway. This turbine blade uses the S826 airfoil series issued by NREL (National Renewable Energy Laboratory), where the rotor diameter is $0.944 \mathrm{~m}$ (Krogstad \& Eriksen, 2013). Single rotor peak performance conditions in Blind Test 4 with freestream wind speed of $11.5 \mathrm{~m} / \mathrm{s}$ as turbine rotor operating conditions in this study (Bartl \& Sætran, 2017).

The single rotor with previous freestream wind speed of $11.5 \mathrm{~m} / \mathrm{s}$ in Blind Test 4 was reduced to the wind speed of $6 \mathrm{~m} / \mathrm{s}$ at the same peak performance is TSR $=6$ with rotor radius of $0.472 \mathrm{~m}$. Meanwhile, the numerical model used is a model with only $1 / 3$ of the domain using periodicity assumptions on the cross-section of the interface by defining it as an interface, the numerical model is then rotated $120^{\circ}$ for $1 / 3$ domain solution with boundary conditions in Fig. 1 to Fig. 2 and Table 1. Inflow velocity $6 \mathrm{~m} / \mathrm{s}$, the turbulent intensity of 5\% and turbulent viscosity ratio by 10 .

Figure 1. Computing domain of CRWT.
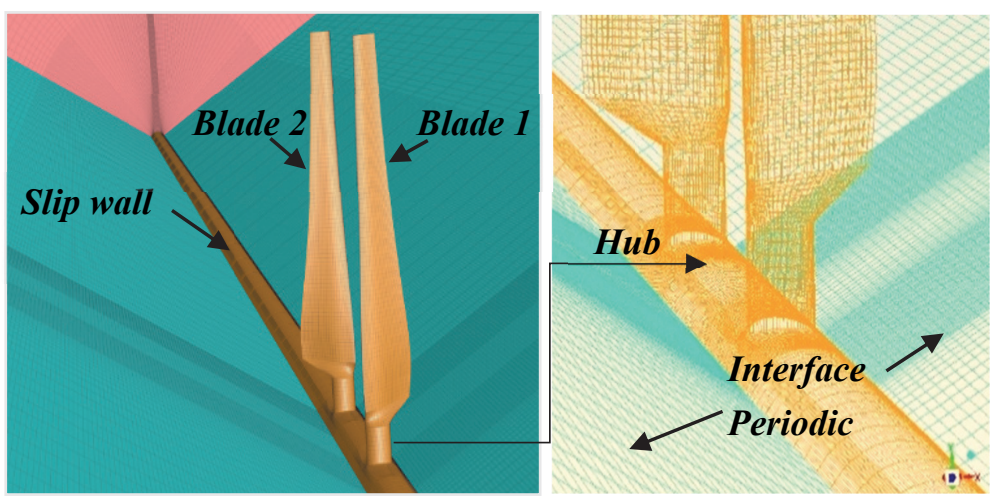

Figure 2. Interface periodic, slip wall, and non-slip condition for blade 1 and blade 2 wall

Table 1. Boundary conditions.

\begin{tabular}{ll}
\hline \multicolumn{1}{c}{ Boundary } & \multicolumn{1}{c}{ Imposed boundary conditions } \\
\hline Inlet & Freestream velocity $6 \mathrm{~m} / \mathrm{s}$ \\
Outlet & Pressure outlet 1 atm
\end{tabular}


Blade (wall)

Front Rotor Diameter $\left(\mathrm{D}_{1}\right)$

Interface periodic

Slip wall

Wall top

Turbulent Intensity

Turbulent viscosity ratio
Non-slip condition

$0.944 \mathrm{~m}$

Periodic (rotational)

Non-slip conditions

Specified shear stress to zero

$5 \%$

10

\subsection{CRWT Parameters and Mesh Quality on CFD Simulation Models}

The meshing decomposition is used by Gambit software with information on the number of mesh elements and mesh quality in Table 2. The meshing quality is stated in several methods including mesh skewness from 0 to 1 and aspect ratio $(<1000)$ from the single rotor CFD simulation model and CRWT can be seen in Table 2 . The density of the mesh around the blade surface is made the same for each variation of the rotor diameter and rotor axial distance to avoid numerical error in the simulation.

Table 2. CRWT parameters and mesh quality on CFD simulation models.

\begin{tabular}{|c|c|c|c|c|c|c|c|}
\hline \multirow{2}{*}{$\begin{array}{l}\text { Blade } \\
\text { models }\end{array}$} & \multirow{2}{*}{$\begin{array}{l}\text { Diameter } \\
\text { ratio, } D_{1} / \mathrm{D}_{2}\end{array}$} & \multirow{2}{*}{$\begin{array}{l}\text { Distance } \\
\text { ratio, } \mathrm{L} / \mathrm{D}_{1}\end{array}$} & \multirow{2}{*}{$\begin{array}{l}\text { Number of mesh } \\
\text { elements (hex) }\end{array}$} & \multicolumn{3}{|c|}{ Mesh skewness, \% } & \multirow{2}{*}{$\begin{array}{c}\text { Mesh aspect ratio, \% } \\
>200\end{array}$} \\
\hline & & & & $>0.35$ & $>0.6$ & $>0.9$ & \\
\hline SRWT & & & $1,340,820$ & 3.26 & 0.51 & 0.01 & 3.69 \\
\hline $\mathrm{DR}_{1} \mathrm{Z}_{1}$ & & 0.25 & $2,917,800$ & 8.59 & 1.51 & 0.01 & 0.03 \\
\hline $\mathrm{DR}_{1} \mathrm{Z}_{2}$ & 0.50 & 0.50 & $3,557,160$ & 4.50 & 0.55 & 0.01 & 0.03 \\
\hline $\mathrm{DR}_{1} \mathrm{Z}_{3}$ & & 0.75 & $3,729,960$ & 4.36 & 0.54 & 0.01 & 0.02 \\
\hline $\mathrm{DR}_{2}$ & 0.75 & 0.25 & $2,147,157$ & 8.31 & 0.60 & 0.01 & 0.59 \\
\hline $\mathrm{DR}_{3} \mathrm{Z}_{1}$ & & 0.20 & $2,088,090$ & 5.18 & 0.63 & 0.01 & 2.87 \\
\hline $\mathrm{DR}_{3} \mathrm{Z}_{2}$ & & 0.25 & $2,197,440$ & 4.98 & 0.62 & 0.01 & 1.86 \\
\hline $\mathrm{DR}_{3} \mathrm{Z}_{3}$ & & 0.30 & $2,088,090$ & 5.60 & 0.66 & 0.01 & 2.87 \\
\hline $\mathrm{DR}_{3} \mathrm{Z}_{4}$ & 100 & 0.40 & $2,513,340$ & 4.57 & 0.62 & 0.01 & 2.19 \\
\hline $\mathrm{DR}_{3} \mathrm{Z}_{5}$ & 1.00 & 0.50 & $2,719,890$ & 4.41 & 0.56 & 0.01 & 2.01 \\
\hline $\mathrm{DR}_{3} \mathrm{Z}_{6}$ & & 0.75 & $3,254,490$ & 4.02 & 0.54 & 0.01 & 1.50 \\
\hline $\mathrm{DR}_{3} \mathrm{Z}_{7}$ & & 1.00 & $3,764,790$ & 3.78 & 0.53 & 0.01 & 1.29 \\
\hline $\mathrm{DR}_{3} \mathrm{Z}_{9}$ & & 1.50 & $3,315,240$ & 4.02 & 0.57 & 0.01 & 1.23 \\
\hline $\mathrm{DR}_{4}$ & 1.25 & 0.25 & $2,235,573$ & 6.88 & 0.64 & 0.01 & 3.06 \\
\hline $\mathrm{DR}_{5}$ & 1.50 & 0.25 & $2,247,804$ & 4.49 & 0.54 & 0.01 & 3.42 \\
\hline
\end{tabular}

\section{Results}

\subsection{Validation of the Numerical Model}

The numerical model validation is done by calculating the errors that occur from the single rotor performance by CFD simulation results on the results of the BT4 (Blind Test 4) experiment. Turbine power coefficient or $\mathrm{C}_{\mathrm{P}}$ as rotor aerodynamic performance is the result of the division between the output power produced by the rotor or $\mathrm{P}_{\text {out }}$ and the total power in wind flow or $\mathrm{P}_{\text {in }}$ for the given rotor sweep area or A as in Eq. 3.

$$
C_{P}=\frac{P_{o u t}}{P_{i n}}
$$

with, $P_{i n}=0,5 \rho A V_{0}^{3}$

where $\mathrm{A}$ is the rotor sweep area with the largest diameter, $\rho$ is the air density, and $\mathrm{V}_{0}$ is the freestream wind speed. The power coefficient is a non-linear function of the blade tip speed ratio or $\lambda$ or tip speed ratio (TSR), where TSR depends on wind speed and shaft rotation speed where $\mathrm{R}$ is the rotor radius and $\omega$ is the rotor angular velocity. The TSR for a single rotor is calculated based on Eq. 4. 


$$
\lambda=\frac{R \omega}{V_{0}}
$$

The next test is how the characteristics of a single rotor wind turbine at lower freestream wind speed conditions by maintaining the same TSR value of the turbine operation (Make \& Vaz, 2015). Decreasing the inflow velocity (freestream) with the same TSR which directly decreases the Reynolds or Re number scale (Burmester et al., 2016) as in Eq. 5. If the resultant velocity of the blade tip is $V_{R}$, kinematic viscosity or $\mu$, and the flow density is $\rho$, then the Reynolds number in the blade tip can be calculated as follows:

$$
R_{e}=\frac{\rho V_{R} c}{\mu}
$$

CFD simulations on a single rotor or CRWT were carried out by lowering the rotor rotation from $1396 \mathrm{rpm}$ (inflow velocity of $11.5 \mathrm{~m} / \mathrm{s}$ at TSR $=6$ with Reynolds number at tip blade of $1.24 \times 10^{5}$ ) to $728 \mathrm{rpm}$ (inflow velocity of 6.0 $\mathrm{m} / \mathrm{s}$ at the same TSR with Reynolds number at tip blade of $6.47 \times 10^{4}$ ).

The prediction results of the single rotor wind turbine performance from CFD simulation through a decrease in inflow velocity are illustrated by the relationship between the power coefficient and tip speed ratio (TSR) in Fig. 3. The rotor performance at both inflow velocity shows a value close enough to the experimental results especially on the TSR value of 4 to 7 . The maximum power coefficient occurs at TSR $=6$ with the lowest error value, that is, for both inflow velocity $6 \mathrm{~m} / \mathrm{s}$ and $11.5 \mathrm{~m} / \mathrm{s}$ is $4.27 \%$ and $3.43 \%$. This result shows that the error that occurs from the CFD simulation model at wind speeds of $6 \mathrm{~m} / \mathrm{s}$ and $11.5 \mathrm{~m} / \mathrm{s}$ is relatively low with an error difference of $0.84 \%$. However, the prediction results on high TSR tend to be lower and vice versa on low TSR tend to be higher than the experimental results (Bartl \& Sætran, 2017).

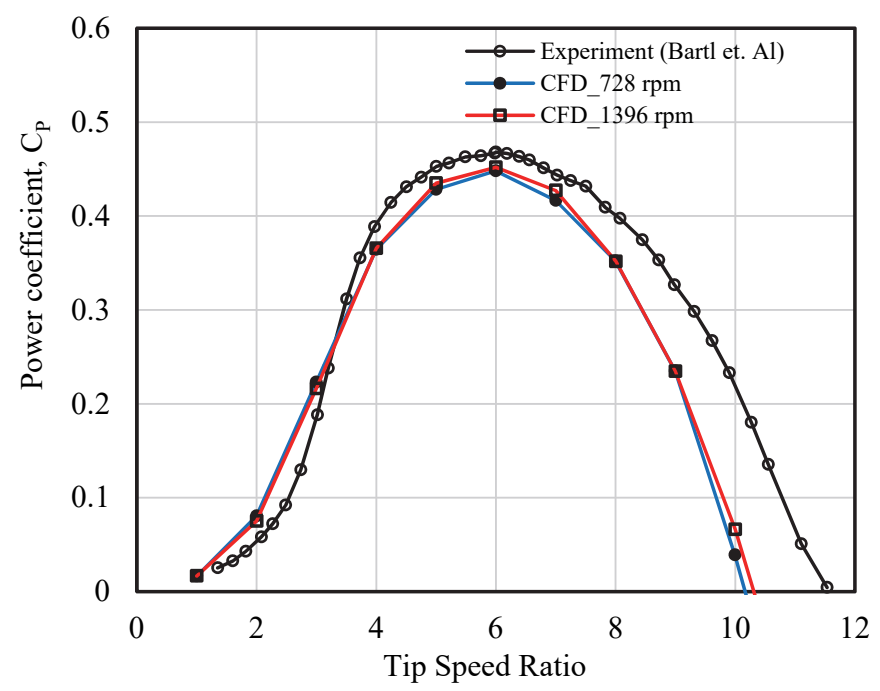

Figure 3. The relationship between power coefficient and tip speed ratio of the single rotor from current CFD simulation versus experiment by Bartl \& Sætran (2017)

The separation of flow on the blade surface at a low TSR through the realizable turbulent k- $\varepsilon$ model cannot be predicted well, especially for flow on the surface with adverse pressure gradient conditions. Hallanger \& Sand (2013) show that the boundary layer on the blades separates and stalling occurs on low TSR. It can be decreasing the ratio between lift and drag and also lower the power coefficient that it is not predicted well by the k- $\varepsilon$ model. Prediction results on low TSR show turbine rotor performance are higher than experimental results. This is understandable because in a low TSR with a low Reynolds number, the realizable turbulent k- $\varepsilon$ model tends to widen from the true value. However, the numerical error displayed is relatively small so that this simulation model can be applied in the analysis of the performance of a single rotor wind turbine and CRWT.

The separation that occurs along the blade surface develops faster and stronger as the TSR decreases. This phenomenon causes the blade to experience a stall which results in an increase in drag in the rotor so that the generated torque decreases rapidly until the $\mathrm{TSR}=1$ torque is close to zero. Whereas in the high TSR (TSR $>7$ ) the rotor tends to experience a decrease in performance as shown in Fig. 3 until the TSR $=10$ decreases rapidly and reaches negative torque at the TSR $>11$. The negative torque shows that the rotor in the TSR is in the condition of 
a propeller. The simulation results of CFD on high TSR tend to be lower than the experimental results.

\subsection{Result of Numerical Simulation}

\subsubsection{Effect of Tip Speed Ratio on the CRWT Performance}

The aerodynamic performance of counter-rotating or CRWT wind turbine rotors has been studied on the tip speed ratio (TSR) between 1 to 10 with variations in rotor diameter ratios and distance ratios. The study through CFD simulation was carried out at a constant rotation ratio, which means that the front and rear rotor rotation is maintained constant with a rotation ratio of $\mathrm{n}_{2} / \mathrm{n}_{1}=0.6$ ( $\mathrm{n}_{1}$ is front rotor rotation and $\mathrm{n}_{2}$ is rear rotor rotation), in accordance with research conducted by Lee et al. (2012). Meanwhile, the TSR variable is obtained from the variation of inflow velocity (freestream) for each TSR equals to the single rotor condition. Simulation parameters such as boundary conditions, solution method, and control solution, as well as mesh density around the blade surface, is made the same for each rotor diameter variation and axial distance to avoid numerical error in the simulation, while the convergent criteria for all parameters simulated by monitoring residual values are $10^{-6}$.

The improvement of CRWT's aerodynamic performance against SRWT from CFD simulation using rear rotor configurations of blade models in Table 2 was obtained in the range of tip speed ratio from 1 to 10 . It is about 15 times 10 simulated data for CRWT and 10 for SRWT. Turbine rotor performance is shown in two conditions, first the turbine rotor performance at each tip speed ratio or $\mathrm{C}_{\mathrm{P}}$ and second the total power coefficient or $\mathrm{C}_{\mathrm{P} \text {-tot }}$ for the TSR range from 1 to 10. Increased front rotor, rear rotor, and CRWT power coefficients in the form of normalization of a single rotor from the results of CFD simulation, namely $\mathrm{C}_{\mathrm{P}} / \mathrm{C}_{\mathrm{P}-\mathrm{SRWT}}$ against tip speed ratio. Meanwhile, the total power coefficient is also expressed in the form of normalization by $\mathrm{C}_{\text {P-tot }} / \mathrm{C}_{\text {P-tot(SRWT). }}$ CRWT tip speed ratio $\left(\lambda_{R}=\lambda_{1}+\lambda_{2}\right)$ is the relative TSR calculated based on the ratio of the relative velocity in the blade tip between the front rotor and the rear rotor to the freestream wind speed (Usui et al., 2012). The total power coefficient of the turbine rotor or $\mathrm{C}_{\mathrm{P} \text {-tot }}$ is the value obtained from the area formed by the power coefficient curve and TSR with an integral approach to the second order polynomial function curve.

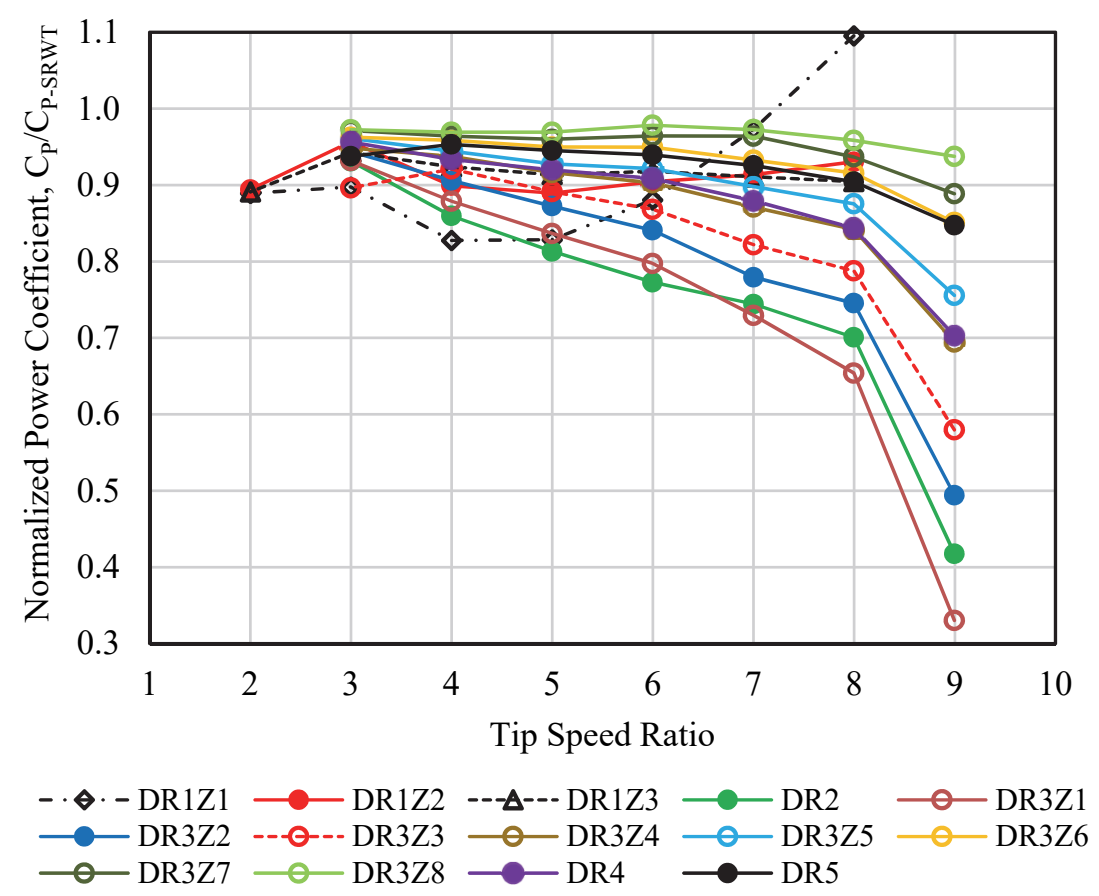

Figure 4. Normalized power coefficients of the front rotor to SRWT through rear rotor configurations using rotor diameter ratio and distance ratio against TSR.

Fig. 4 shows various rear rotor parameters for each addition of TSR from results of normalizing the front rotor power coefficients to SRWT. The normalized power coefficient of the front rotor showed a strong influence of the rear rotor configuration with the addition of TSR. The rear rotor position that is close enough to the front rotor and with the position of the rear rotor blade tip that is close to parallel to the front rotor blade tip can worsen the front rotor performance with the addition of TSR. Conversely, for CRWT with rotor diameter ratios of $D_{1} / D_{2}=0.5$ and 
$\mathrm{D}_{1} / \mathrm{D}_{2}=1.5$, the position of the blade tip is quite far apart in the CRWT with a sufficiently large rotor distance ratio of $\mathrm{L} / \mathrm{D}_{1}>0.5$, the front rotor performance is better and tends to be more gentle with the addition of TSR.

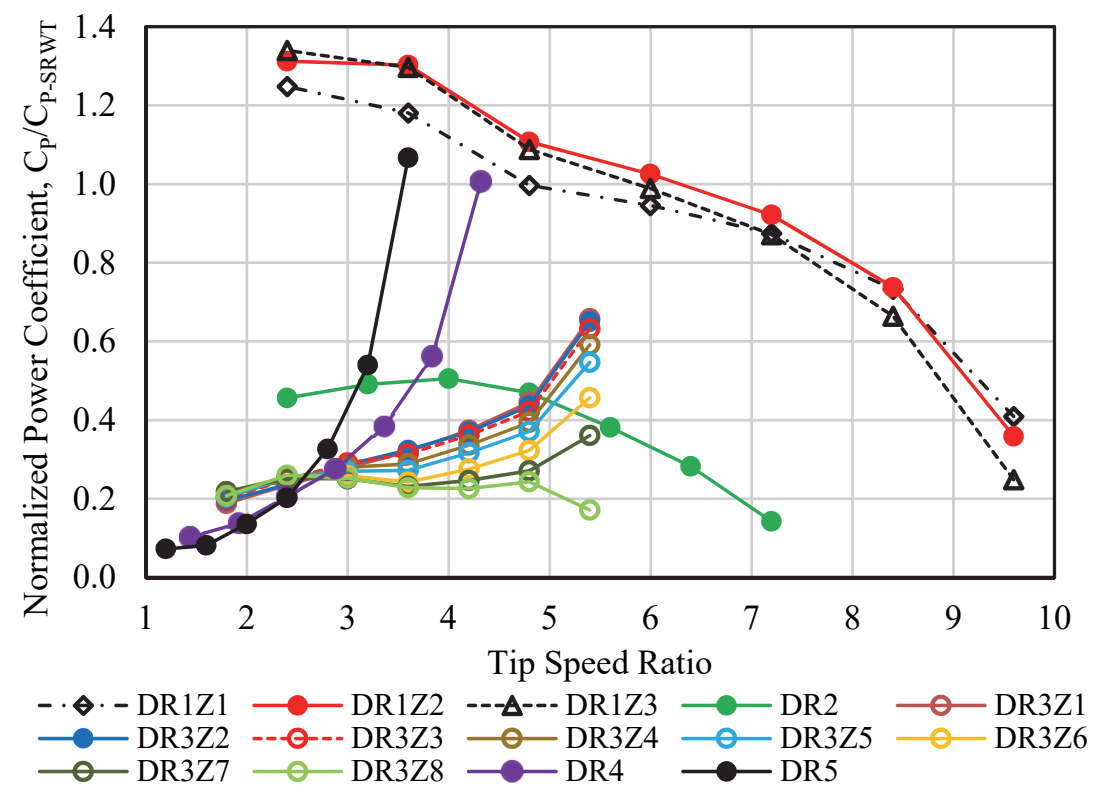

Figure 5. Normalized power coefficient of the rear rotor to SRWT through rear rotor configurations using rotor diameter ratio and distance ratio against TSR.

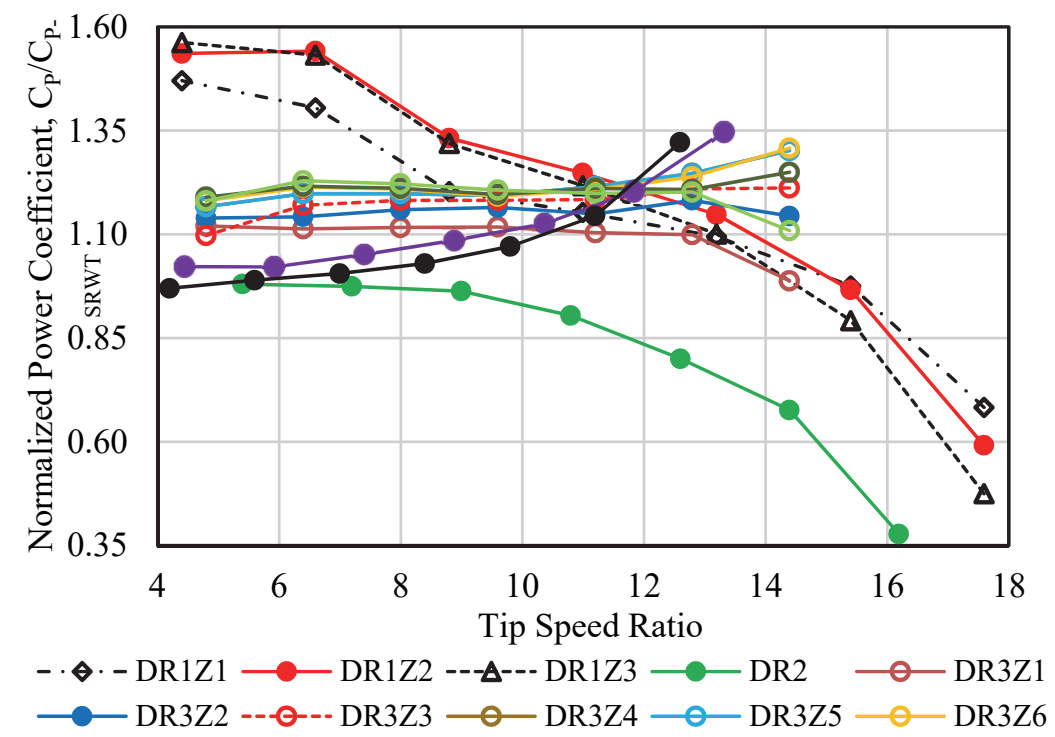

Figure 6. Normalized power coefficient of the combined rotor or CRWT to SRWT through rear rotor configurations using rotor diameter ratio and distance ratio against TSR.

Fig. 5 shows various rear rotor parameters for each addition of TSR from results of normalizing the rear rotor power coefficients to SRWT. The normalized power coefficient of the rear rotor to the single rotor showed a more strong influence of the rear rotor configuration with opposite results to the addition of TSR. CRWT with rotor diameter ratios of $\mathrm{D}_{1} / \mathrm{D}_{2}<1.0$ in Fig 5 shows that values tend to be high on low TSR but instead tend to decrease with the addition of TSR. On the contrary rear rotor with rotor diameter ratio of $\mathrm{D}_{1} / \mathrm{D}_{2} \geq 1.0$ shows the normalized power coefficients which tend to be low on low TSR but instead tends to increase with the addition of TSR. Rear rotor configuration with a fairly wide rotor distance, the normalized power coefficients tend decreases on higher TSR. The results of the combined CRWT or combination of front and rear rotor power coefficient normalization 
or CRWT relatively have the same trend as the rear rotor, but in CRWT with the rotor diameter ratio of D1/D2=1.0, the normalized power coefficients are relatively constant with the addition of TSR (see Fig. 6).

\subsubsection{Effect of Rear Rotor Configurations on the CRWT Performance}

Fig. 7 shows the average results of the normalization of the front rotor, rear rotor and combined that two rotors of CRWT power coefficient on SRWT from rear rotor configuration parameters. The effect of adding rotor diameter ratios and rotor distance ratios can increase the average normalized power coefficients of the front rotor and combined rotor or CRWT, but on the contrary, the rear rotor has decreased. A larger average normalized power coefficient of CRWT to SRWT takes place at a rotor diameter ratio or $\mathrm{D}_{1} / \mathrm{D}_{2}=1.0$ with $\mathrm{L} / \mathrm{D}_{1}=0.75$ by 1.221 . It means that the average power coefficient of CRWT increased by $22.1 \%$ to the SRWT for the given TSR range.

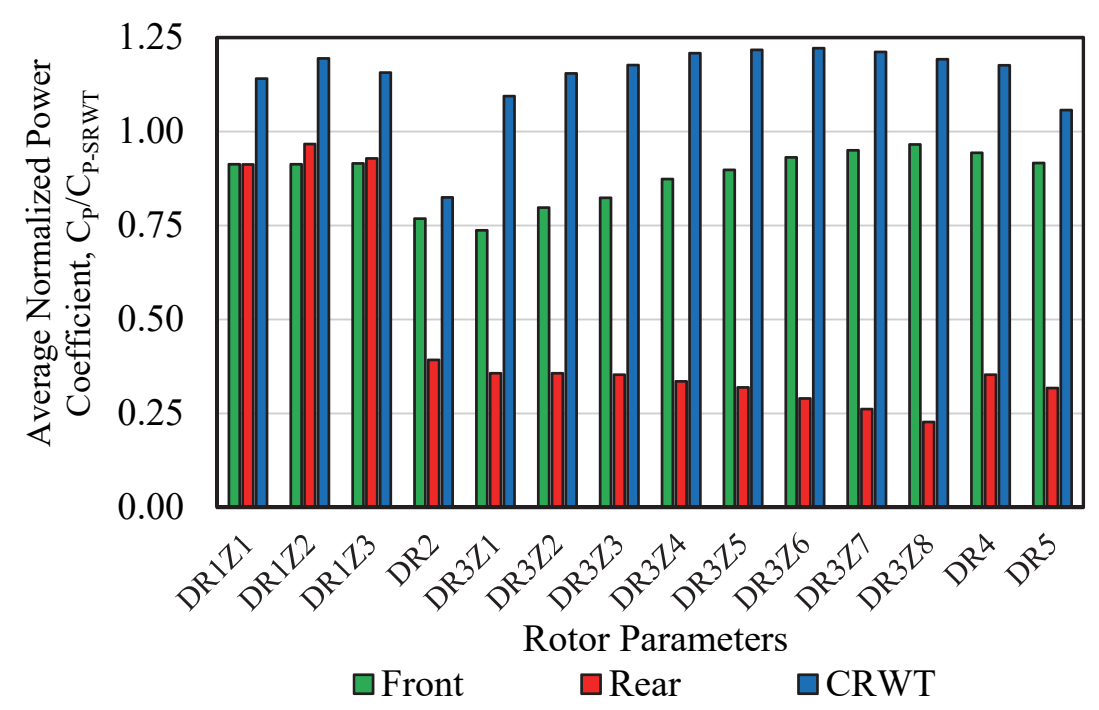

Figure 7. Average normalized power coefficients of the front rotor, rear rotor and CRWT to SRWT through variations in diameter ratio and the ratio of rotor distance against TSR

Fig. 8 and Fig. 9 each show the normalization of total power coefficient or $\mathrm{C}_{\mathrm{P} \text {-tot }}$ of the front rotor, rear rotor, and combined rotor (CRWT) on the variation in diameter ratio and distance ratio. The performance of CRWT was showing in the relationship of power coefficient to tip speed ratio with rotor diameter ratio of $D_{1} / D_{2}=0.5$ until $\mathrm{D}_{1} / \mathrm{D}_{2}=1.5$ at distance ratio of $\mathrm{L} / \mathrm{D}_{1}=0.25$. Furthermore, in the same way for all diameter ratio variables and distance ratios from Table 2, a graph of the relationship of power coefficients to TSR is used to calculate total power coefficient or $C_{P \text {-tot }}$. The CRWT performance from the CFD results for a diameter ratio of $D_{1} / D_{2}=0.5$ indicates that the front rotor operates on a wider TSR range, this results in combined performance (CRWT) occurring in the wider TSR range. On the contrary with the addition of the diameter ratio, however, the front rotor performance is getting better and approaching the performance of a single rotor turbine. The performance of the rear rotor with the addition of the diameter ratio tends to operate in a narrower TSR range so that the operating area of the CRWT is also relatively narrow. The increase and decrease of the front rotor and rear rotor power coefficient provide an increase in combined performance or CRWT to SRWT with increasing the axial distance of the rotor, this is consistent with the research conducted by Lee et al. (2013). Normalization results of the highest total power coefficient or $\mathrm{C}_{\mathrm{P} \text {-tot }}$ occur at a diameter ratio of $\mathrm{D}_{1} / \mathrm{D}_{2}=0.5$ by 2.103 . 


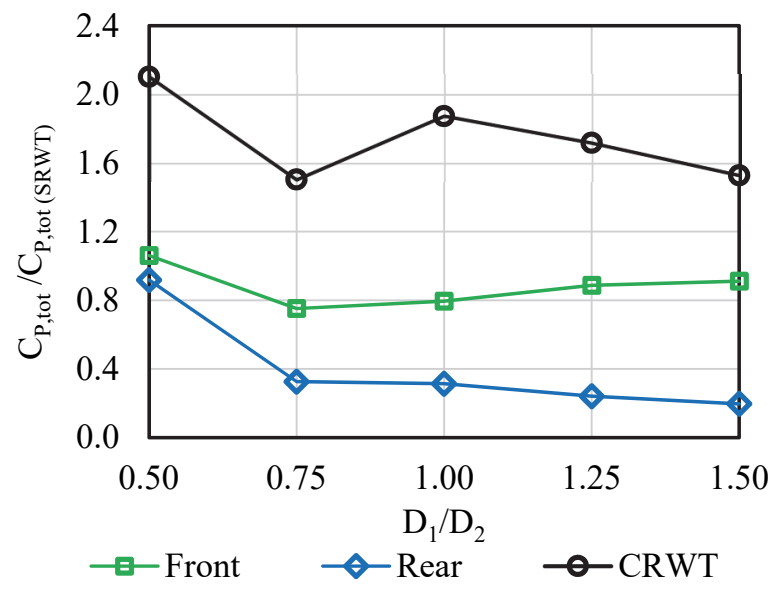

Figure 8. Normalized total power coefficient of CRWT against SRWT through variations in rotor diameter ratios for $\mathrm{L} / \mathrm{D}_{1}=0.25$

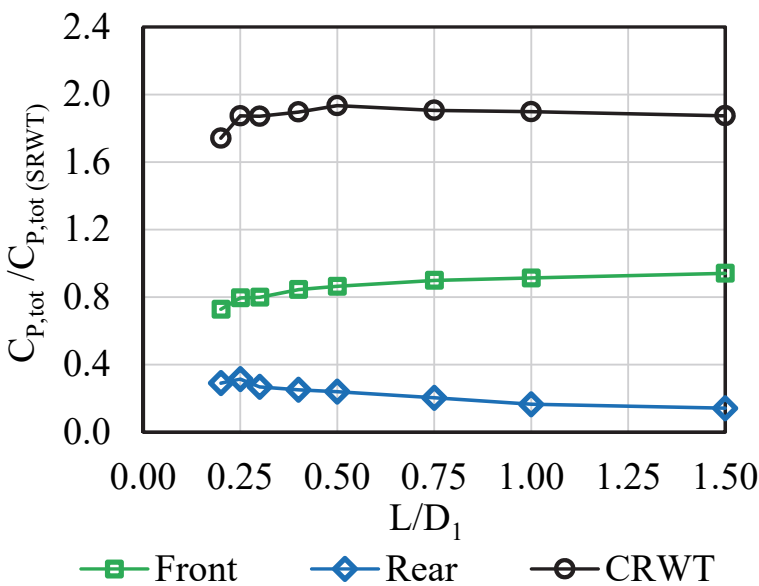

Figure 9. Normalized total power coefficient of CRWT on SRWT through variations in the rotor axial distance ratios for $\mathrm{D}_{1} / \mathrm{D}_{2}=1.0$

The increase in the total power coefficient of CRWT against SRWT through variations in rotor distance ratios with a diameter ratio of $\mathrm{D}_{1} / \mathrm{D}_{2}=1.0$ is the highest in the ratio of distance $\mathrm{L} / \mathrm{D}_{1}=0.5$ or $\mathrm{DZ} 5$, which is 1.934 . If the axial distance of the two rotors continues to be widened, the flow interference by the rear rotor has no effect on the front rotor performance such as the research conducted by Bartl \& Sætran, (2017) with axial distances of 2.77D, 5.18D, and 9D, hence the power coefficient rear rotors will increase again with increasing rotor distance. This phenomenon can be explained for the CRWT rotor distance that is close enough, the interference flow from the rear rotor and the acceleration of recovery of the flow velocity of the front rotor are a very strong influence on the performance of the two rotors. When the rear rotor position is far enough from the front rotor, the flow disturbance from the rear rotor decreases and at the same time the front rotor performance tends to be stable and equal to SRWT.

\section{Discussion}

\subsection{Flow Visualization of the CRWT with $T S R_{1}=3,6$, and 10}

Visualization of flow in wind turbine rotors conducted by Zhang et al. (2012), Massouh and Dobrev (2014), and Tran et al. (2015) was adopted to describe the flow field phenomenon around the CRWT blade or near wake of counter-rotating wind turbine through various rear rotor configurations. The flow pattern with the presence of the rear rotor for the CRWT case has the effect of non-uniform flow both before and after the rear rotor. The formation of a high-velocity gradient in the area around the blade tip and blade hub of the front rotor and rear rotor gives the strong vortex in these areas. 
Fig. 10 and Fig. 11 show axial velocity contour around the hub and tip blade on the plane perpendicular to the 50\% chord tip line of SRWT and rear rotor of CRWT with $\mathrm{D}_{1} / \mathrm{D}_{2}=1.0$ and $\mathrm{L} / \mathrm{D}_{1}=0.25$ at three different TSR of 3,6 , and 10. The axial velocity contour around the blade of SRWT at TSR=3 shows that separation occurs in the hub and tends to disappear with increasing TSR (Hallanger \& Sand, 2013). The separation on the hub blade of rear rotor occurs to be growing larger at TSR $=3$, even there is still visible separation at TSR $=6$. Figure 12 shows that the magnitude and direction of the velocity vector at $\mathrm{TSR}=3$ in the rear rotor tends to move towards in radial direction higher than the SRWT condition. This occurs because the rear rotor operated on the wake of the front rotor with lower axial velocity certainly increases the rear rotor angle of attack. Meanwhile, around SRWT and CRWT blade tip, there is an increase in velocity gradient and tends to increase in the radial direction with increasing TSR. This is shown in TSR $=10$ rear rotor blade tip, velocity gradients and resultant of velocity are stronger than SRWT conditions (see Fig. 13). The stronger the velocity gradient and resultant of velocity to the radial direction, the more increase the centrifugal forces and thrust which can reduce torque output (Mo \& Lee, 2012).

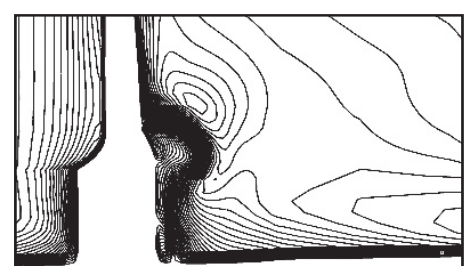

a) $\mathrm{TSR}=3(\mathrm{SRWT})$

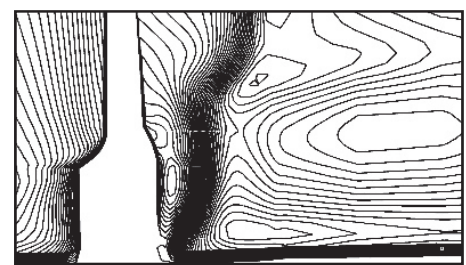

d) $\mathrm{TSR}_{1}=3$ (rear)

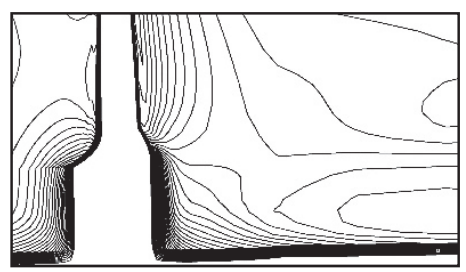

b) $\mathrm{TSR}=6(\mathrm{SRWT})$

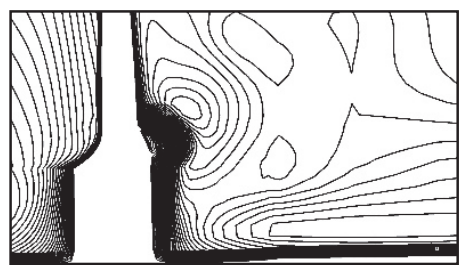

e) $\mathrm{TSR}_{1}=6$ (rear)

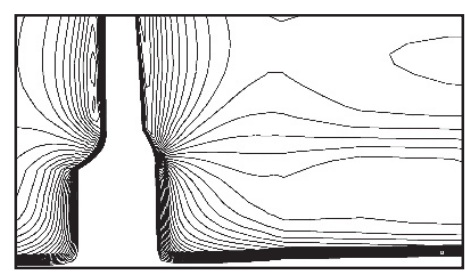

c) $\mathrm{TSR}=10(\mathrm{SRWT})$

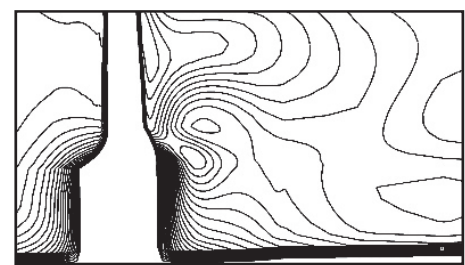

f) $\mathrm{TSR}_{1}=10$ (rear)

Figure 10. Axial velocity contour around the hub on the plane perpendicular to the $50 \%$ chord tip line of SRWT and rear rotor of CRWT $\left(\mathrm{D}_{1} / \mathrm{D}_{2}=1.0 ; \mathrm{L} / \mathrm{D}_{1}=0.25\right)$ with $\mathrm{TSR}_{1}=3,6$, and 10

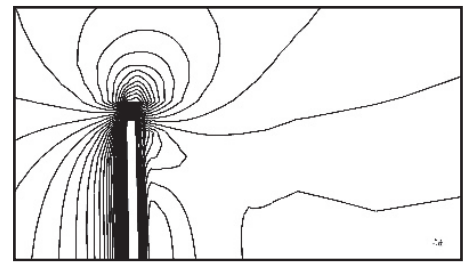

a) $\mathrm{TSR}=3$ (SRWT)

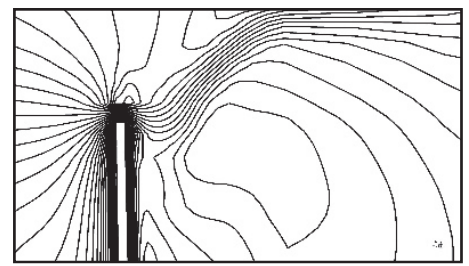

d) $\mathrm{TSR}_{1}=3$ (rear)

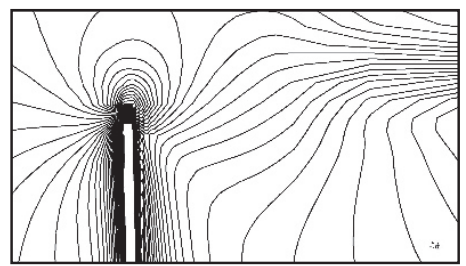

b) $\mathrm{TSR}=6(\mathrm{SRWT})$

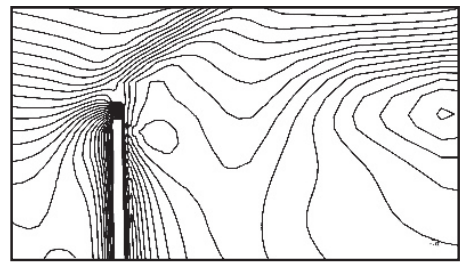

e) $\mathrm{TSR}_{1}=6$ (rear)

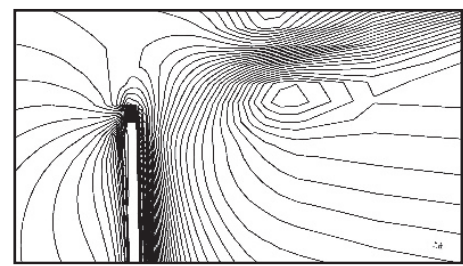

c) $\mathrm{TSR}=10(\mathrm{SRWT})$

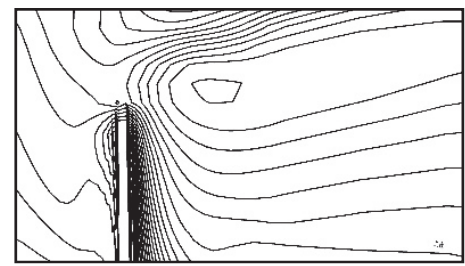

f) $\mathrm{TSR}_{1}=10$ (rear)

Figire 11. Axial velocity contour around the tip on the plane perpendicular to the $50 \%$ chord tip line of SRWT and rear rotor of CRWT $\left(\mathrm{D}_{1} / \mathrm{D}_{2}=1.0 ; \mathrm{L} / \mathrm{D}_{1}=0.25\right)$ with $\mathrm{TSR}_{1}=3,6$, and 10 


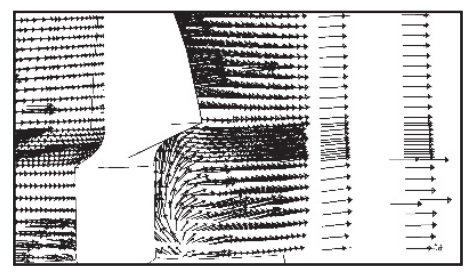

a) $\mathrm{TSR}=3(\mathrm{SRWT})$

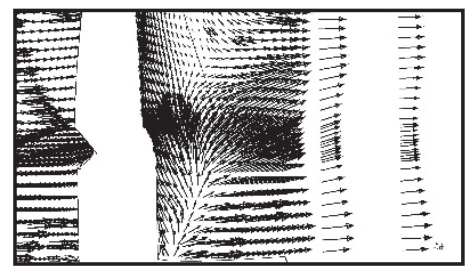

d) $\mathrm{TSR}_{1}=3$ (rear)

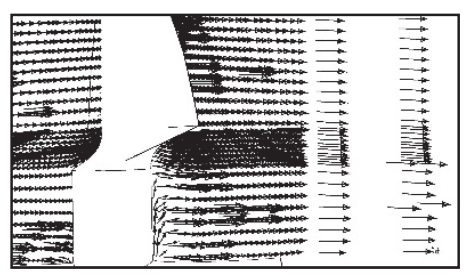

b) $\mathrm{TSR}=6(\mathrm{SRWT})$

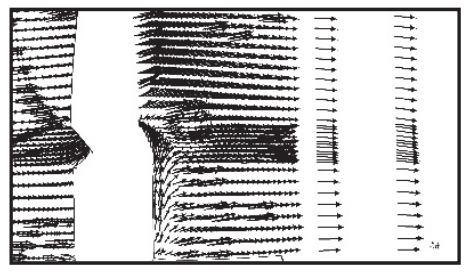

e) $\mathrm{TSR}_{1}=6$ (rear)

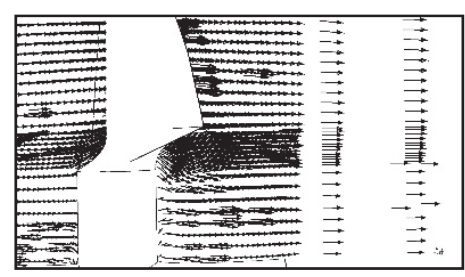

c) $\mathrm{TSR}=10(\mathrm{SRWT})$

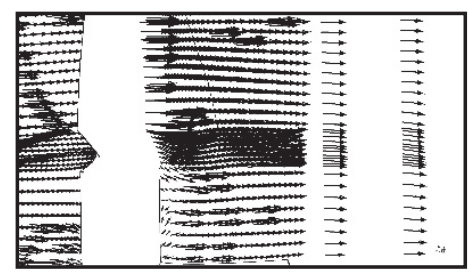

f) $\mathrm{TSR}_{1}=10$ (rear)

Figure 12. Resultant velocity vector around the hub on the plane perpendicular to the $50 \%$ chord tip line of SRWT and rear rotor of CRWT $\left(\mathrm{D}_{1} / \mathrm{D}_{2}=1.0\right.$ and $\left.\mathrm{L} / \mathrm{D}_{1}=0.25\right)$ with $\mathrm{TSR}_{1}=3,6$, and 10

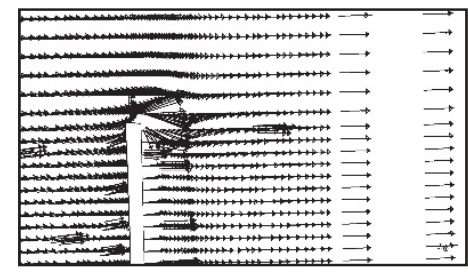

a) $\mathrm{TSR}=3(\mathrm{SRWT})$

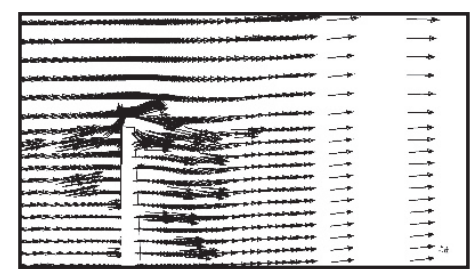

d) $\mathrm{TSR}_{1}=3$ (rear)

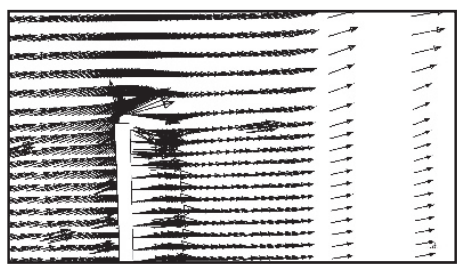

b) $\mathrm{TSR}=6(\mathrm{SRWT})$

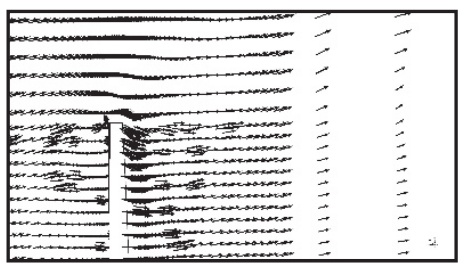

e) $\mathrm{TSR}_{1}=6$ (rear)

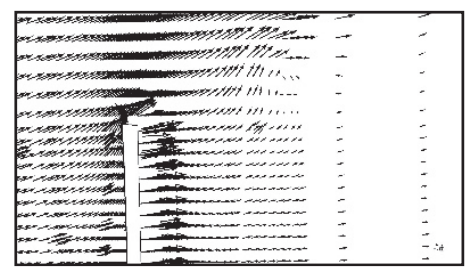

c) $\mathrm{TSR}=10(\mathrm{SRWT})$

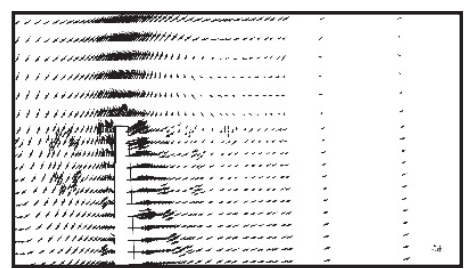

f) $\mathrm{TSR}_{1}=10$ (rear)

Figure 13. Resultant velocity vector around the tip on the plane perpendicular to the $50 \%$ chord tip line of SRWT and rear rotor of CRWT $\left(\mathrm{D}_{1} / \mathrm{D}_{2}=1.0\right.$ and $\left.\mathrm{L} / \mathrm{D}_{1}=0.25\right)$ with $\mathrm{TSR}_{1}=3,6$, and 10

\subsection{Flow Visualization of the Rear Rotor Configuration}

Fig. 14 and Fig. 15 show the axial velocity contour around the hub and tip blade on the plane perpendicular to the $50 \%$ chord tip line of various rear rotor configurations at $\mathrm{TSR}_{1}=3$. The axial velocity contour around the hub blade of rear rotor configurations shows that separation occurs in the hub and tends to disappear with increasing the rotor diameter ratio. Its larger separation on the hub blade of the rear rotor at $D_{1} / D_{2}=1.5$ with $L / D_{1}=0.25$ is caused in CRWT performance of this rear rotor to be lower. The axial velocity contour around the tip blade of rear rotor configurations at $\mathrm{TSR}=10$ shows that there is an increase in the velocity gradient and tends to be increased in the radial direction with decreasing the rotor diameter ratio. Larger velocity gradient on the tip blade at the rear rotor of $\mathrm{D}_{1} / \mathrm{D}_{2}=0.5$ with $\mathrm{L} / \mathrm{D}_{1}=0.25$ is caused in CRWT performance of this rear rotor to be lower.

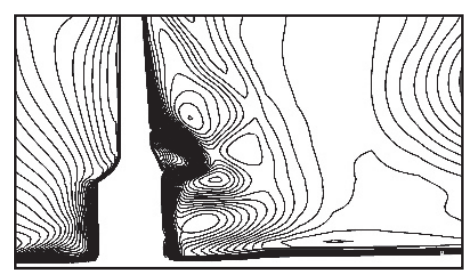

a) $\mathrm{D}_{1} / \mathrm{D}_{2}=0.5 ; \mathrm{L} / \mathrm{D}_{1}=0.25$

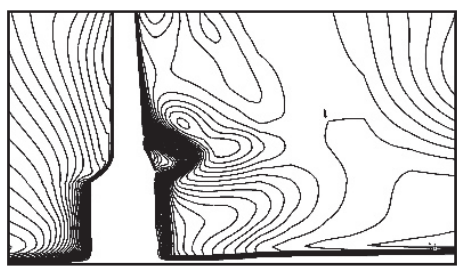

b) $\mathrm{D}_{1} / \mathrm{D}_{2}=0.5 ; \mathrm{L} / \mathrm{D}_{1}=0.5$

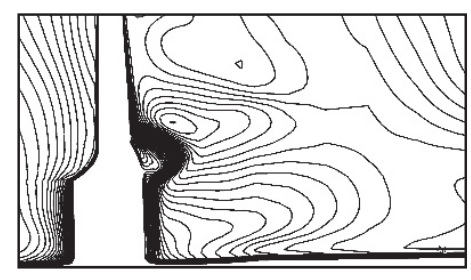

c) $\mathrm{D}_{1} / \mathrm{D}_{2}=0.5 ; \mathrm{L} / \mathrm{D}_{1}=0.75$ 


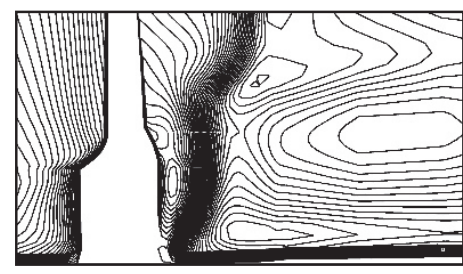

d) $\mathrm{D}_{1} / \mathrm{D}_{2}=1.0 ; \mathrm{L} / \mathrm{D}_{1}=0.25$

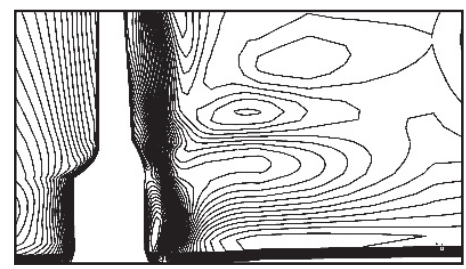

g) $\mathrm{D}_{1} / \mathrm{D}_{2}=0.75 ; \mathrm{L} / \mathrm{D}_{1}=0.25$

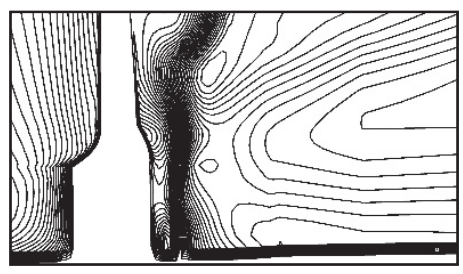

e) $\mathrm{D}_{1} / \mathrm{D}_{2}=1.0 ; \mathrm{L} / \mathrm{D}_{1}=0.5$

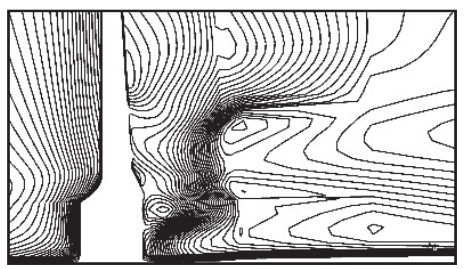

h) $\mathrm{D}_{1} / \mathrm{D}_{2}=1.25 ; \mathrm{L} / \mathrm{D}_{1}=0.25$

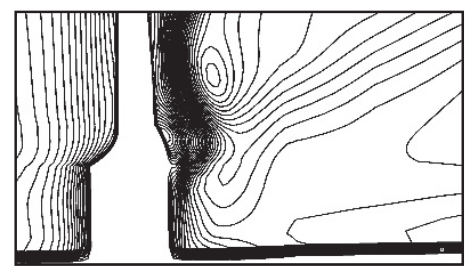

f) $\mathrm{D}_{1} / \mathrm{D}_{2}=1.0 ; \mathrm{L} / \mathrm{D}_{1}=0.75$

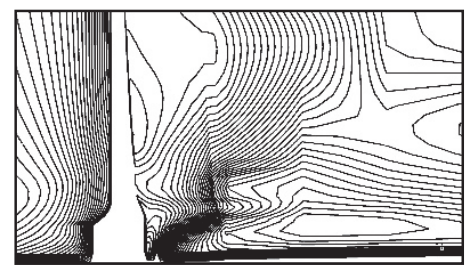

i) $\mathrm{D}_{1} / \mathrm{D}_{2}=1.5 ; \mathrm{L} / \mathrm{D}_{1}=0.25$

Figure 14. Axial velocity contour around the hub on the plane perpendicular to the $50 \%$ chord tip line of various rear rotor configurations at $\mathrm{TSR}_{1}=3$

A high axial velocity drop in this tip area shows a flow with a high-velocity gradient that moves from the tip to the downstream direction. A high-velocity gradient accelerates and strengthens vortex growth in the blade tip (Rosenberg et al., 2014). The higher the TSR, the movement of the vortex tip moves closer to the tip blade which has the effect of increasing the loss of flow in the area. This shows a very strong and continuously increasing flow interference by the rear rotor in the front rotor with a diameter ratio of $\mathrm{D}_{1} / \mathrm{D}_{2}<1.5$ and a larger rotor distance ratio in contrast to the TSR $=3$.

Fig. 16 and Fig. 17 show the resultant velocity vectors around the hub and tip blade on the plane perpendicular to the $50 \%$ chord tip line of various rear rotor configurations at $\mathrm{TSR}_{1}=3$. The resultant velocity vectors around the hub blade of rear rotor configurations are shows that separation occurs with bound vortex near the hub and tends to higher with decreasing the rotor diameter ratio. Its larger bound vortex on the hub blade of the rear rotor at $\mathrm{D}_{1} / \mathrm{D}_{2}=1.5$ with $\mathrm{L} / \mathrm{D}_{1}=0.25$ this increase by resultant velocity vector to the radial direction. The resultant velocity vectors around the blade tip of rear rotor configurations at $\mathrm{TSR}=10$ shows that larger bound vortex takes place and tends to increase in the radial direction with decreasing the rotor diameter ratio. Larger bound vortex on the tip blade at the rear rotor of $D_{1} / D_{2}=0.5$ with $L / D_{1}=0.25$ is caused in CRWT performance of this rear rotor to be lower.

The rear rotor inflow velocity shows a steeper decrease in the ratio of the rotor diameter and almost the entire cross section of the blade. The steep velocity reduction towards the rear rotor with the diameter ratio addition shows the low inflow velocity at the rear rotor, which worsens the rear rotor performance, even though there is an increase in the front rotor performance. In addition, through the addition of the CRWT rotor distance, for example, in the rotor distance ratio of $L / D_{1}=0.5$ and $L / D_{1}=0.75$ with a diameter ratio of $D_{1} / D_{2}=1.0$ indicates a high-velocity decrease after the front rotor and higher again when entering the rear rotor. This causes a decrease in rear rotor performance with the addition of a rotor distance ratio of $\mathrm{L} / \mathrm{D}_{1}=0.5$ and $\mathrm{L} / \mathrm{D}_{1}=0.75$, but the front rotor has improved performance. At a fairly close rotor distance of $\mathrm{L} / \mathrm{D}_{1}=0.25$, the presence of high-velocity inflow by the rear rotor causes a low rate of recovery of wind velocity so that a high decrease in velocity. Whereas, if at $\mathrm{D}_{1} / \mathrm{D}_{2}=0.5$ the distance of the rotor is increased to $L / D_{1}=0.5$, then the rate of recovery of flow velocity becomes high and the interference of flow by the rear rotor decreases. This condition causes the CRWT's front rotor performance with $\mathrm{L} / \mathrm{D}_{1}$ rotor distance $>0.25$ to be better than $\mathrm{L} / \mathrm{D}_{1}=0.25$.

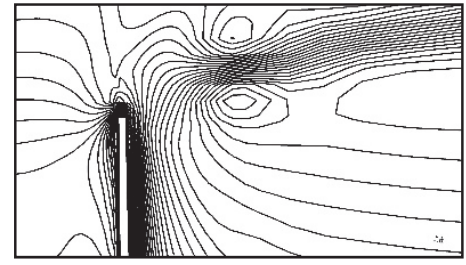

a) $\mathrm{D}_{1} / \mathrm{D}_{2}=0.5 ; \mathrm{L} / \mathrm{D}_{1}=0.25$

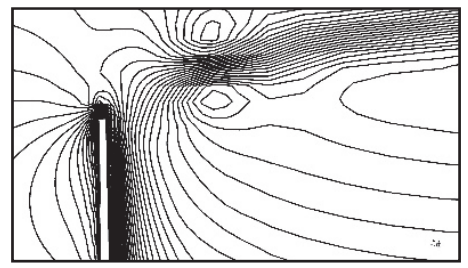

b) $\mathrm{D}_{1} / \mathrm{D}_{2}=0.5 ; \mathrm{L} / \mathrm{D}_{1}=0.5$

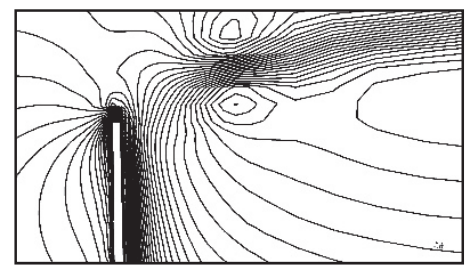

c) $\mathrm{D}_{1} / \mathrm{D}_{2}=0.5 ; \mathrm{L} / \mathrm{D}_{1}=0.75$ 


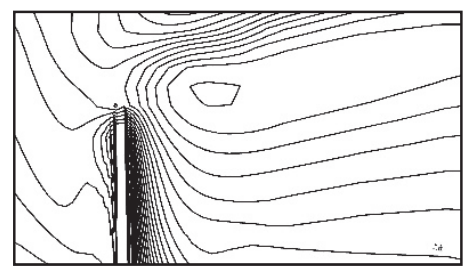

d) $\mathrm{D}_{1} / \mathrm{D}_{2}=1.0 ; \mathrm{L} / \mathrm{D}_{1}=0.25$

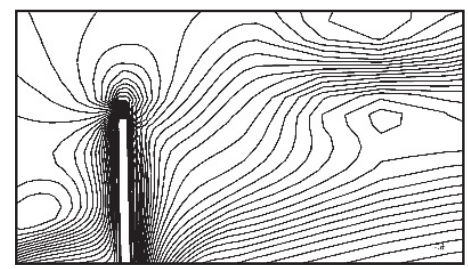

g) $\mathrm{D}_{1} / \mathrm{D}_{2}=0.75 ; \mathrm{L} / \mathrm{D}_{1}=0.25$

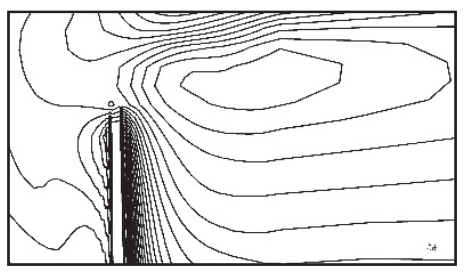

e) $\mathrm{D}_{1} / \mathrm{D}_{2}=1.0 ; \mathrm{L} / \mathrm{D}_{1}=0.5$

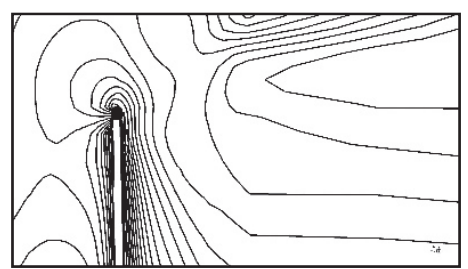

h) $\mathrm{D}_{1} / \mathrm{D}_{2}=1.25 ; \mathrm{L} / \mathrm{D}_{1}=0.25$

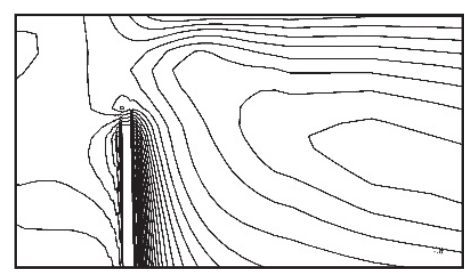

f) $\mathrm{D}_{1} / \mathrm{D}_{2}=1.0 ; \mathrm{L} / \mathrm{D}_{1}=0.75$

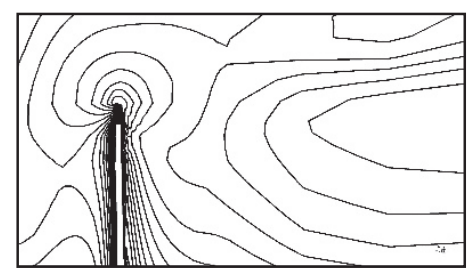

i) $\mathrm{D}_{1} / \mathrm{D}_{2}=1.5 ; \mathrm{L} / \mathrm{D}_{1}=0.25$

Figure 15. Axial velocity contour around the tip on the plane perpendicular to the $50 \%$ chord tip line of various rear rotor configurations at $\mathrm{TSR}_{1}=10$

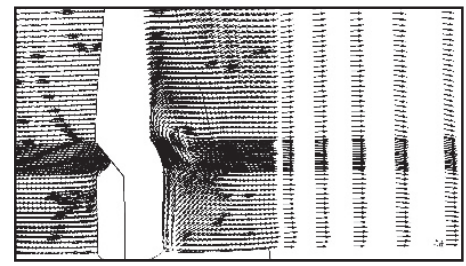

a) $\mathrm{D}_{1} / \mathrm{D}_{2}=0.5 ; \mathrm{L} / \mathrm{D}_{1}=0.25$

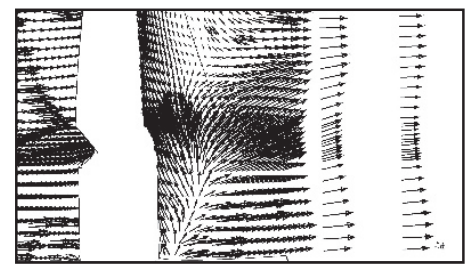

d) $\mathrm{D}_{1} / \mathrm{D}_{2}=1.0 ; \mathrm{L} / \mathrm{D}_{1}=0.25$

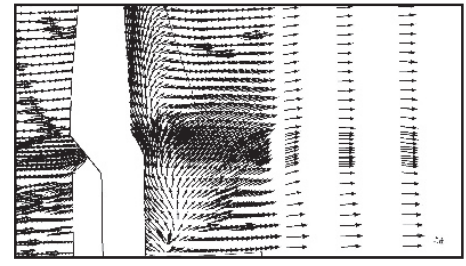

g) $\mathrm{D}_{1} / \mathrm{D}_{2}=0.75 ; \mathrm{L} / \mathrm{D}_{1}=0.25$

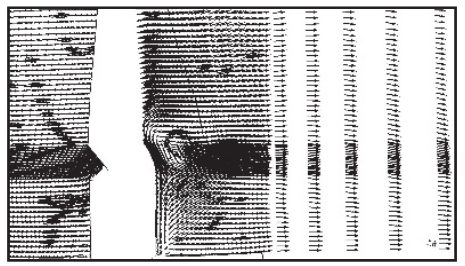

b) $\mathrm{D}_{1} / \mathrm{D}_{2}=0.5 ; \mathrm{L} / \mathrm{D}_{1}=0.5$

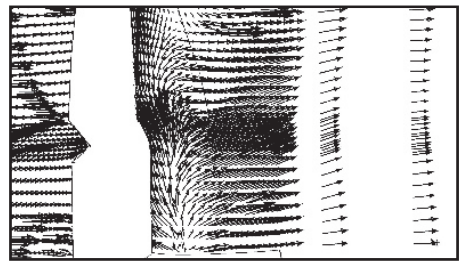

e) $\mathrm{D} 1 / \mathrm{D} 2=1.0 ; \mathrm{L} / \mathrm{D} 1=0.5$

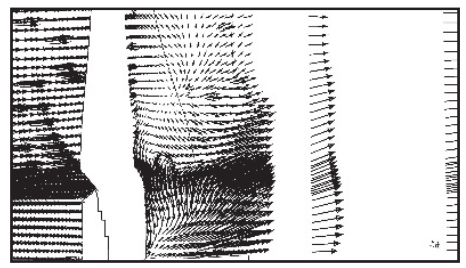

h) $\mathrm{D}_{1} / \mathrm{D}_{2}=1.25 ; \mathrm{L} / \mathrm{D}_{1}=0.25$

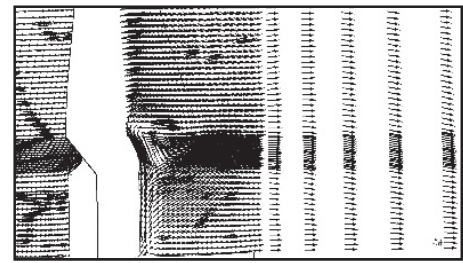

c) $\mathrm{D}_{1} / \mathrm{D}_{2}=0.5 ; \mathrm{L} / \mathrm{D}_{1}=0.75$

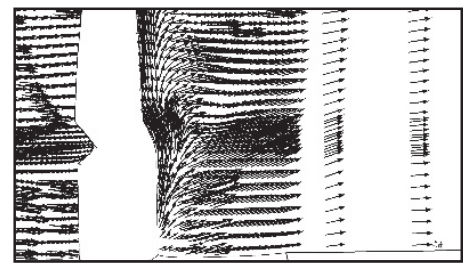

f) $\mathrm{D}_{1} / \mathrm{D}_{2}=1.0 ; \mathrm{L} / \mathrm{D}_{1}=0.75$

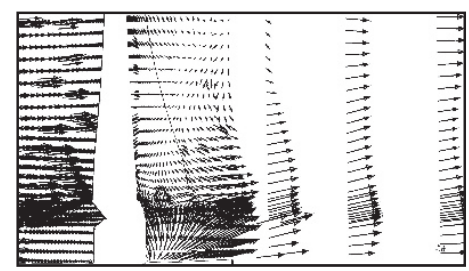

i) $\mathrm{D} 1 / \mathrm{D} 2=1.5 ; \mathrm{L} / \mathrm{D} 1=0.25$

Figure 16. Resultant velocity vector around the hub on the plane perpendicular to the $50 \%$ chord tip line of various rear rotor configurations at $\mathrm{TSR}_{1}=3$

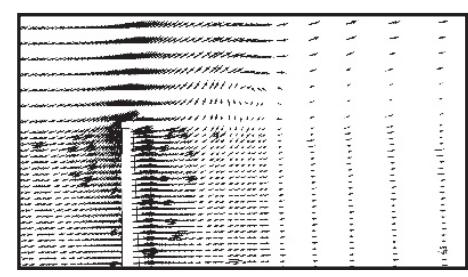

a) $\mathrm{D}_{1} / \mathrm{D}_{2}=0.5 ; \mathrm{L} / \mathrm{D}_{1}=0.25$

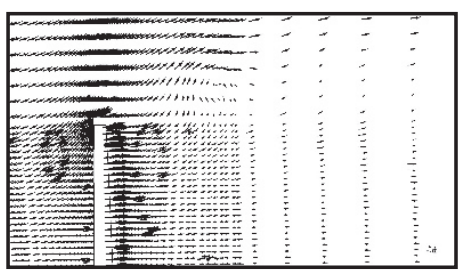

b) $\mathrm{D}_{1} / \mathrm{D}_{2}=0.5 ; \mathrm{L} / \mathrm{D}_{1}=0.5$

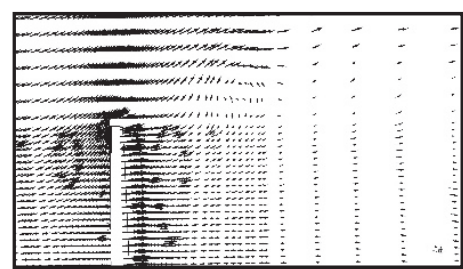

c) $\mathrm{D}_{1} / \mathrm{D}_{2}=0.5 ; \mathrm{L} / \mathrm{D}_{1}=0.75$ 


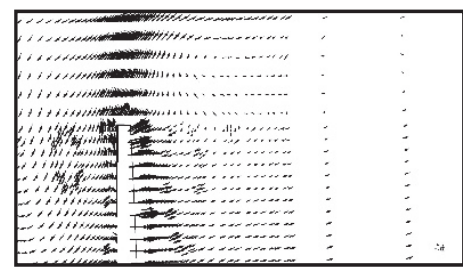

d) $\mathrm{D}_{1} / \mathrm{D}_{2}=1.0 ; \mathrm{L} / \mathrm{D}_{1}=0.25$

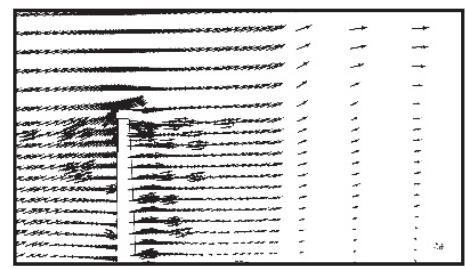

g) $\mathrm{D}_{1} / \mathrm{D}_{2}=0.75 ; \mathrm{L} / \mathrm{D}_{1}=0.25$

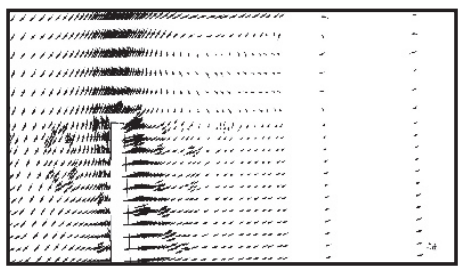

e) $\mathrm{D}_{1} / \mathrm{D}_{2}=1.0 ; \mathrm{L} / \mathrm{D}_{1}=0.5$

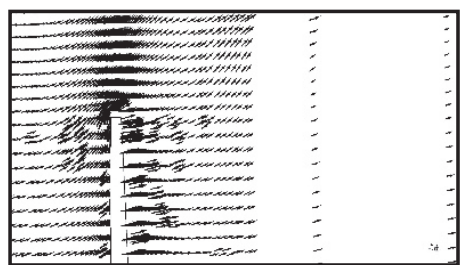

h) $\mathrm{D}_{1} / \mathrm{D}_{2}=1.25 ; \mathrm{L} / \mathrm{D}_{1}=0.25$

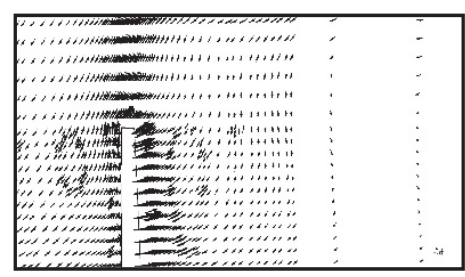

f) $\mathrm{D}_{1} / \mathrm{D}_{2}=1.0 ; \mathrm{L} / \mathrm{D}_{1}=0.75$

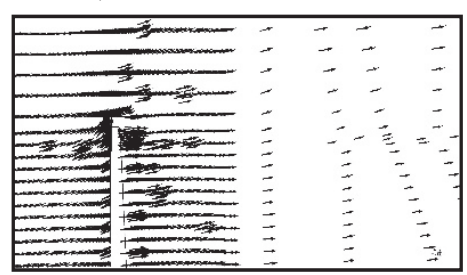

i) $\mathrm{D}_{1} / \mathrm{D}_{2}=1.5 ; \mathrm{L} / \mathrm{D}_{1}=0.25$

Figure 17 . Resultant velocity vector around the tip on the plane perpendicular to the $50 \%$ chord tip line of various rear rotor configurations at $\mathrm{TSR}_{1}=10$

More detailed axial velocity contours show flow fluctuations due to vortex growth in the tip and blade hub after flow through the second rotor. CRWT with a ratio of rotor diameter $\mathrm{D}_{1} / \mathrm{D}_{2}<1.0$, the vortex tip from the front rotor gives an additional vortex in the rear rotor to three places as seen in the ratio of the rotor diameter $D_{1} / D_{2}=0.5$. Whereas separation in the hub area for the ratio of diameter $D_{1} / D_{2} \geq 1.0$ tends to occur, while with the addition of rotor distance, the effect of the vortex tip and hub is more strongly suffered by the rear rotor.

The axial velocity contour around the CRWT with a diameter ratio of $\mathrm{D}_{1} / \mathrm{D}_{2}>0.5$ and the distance of the rear rotor that is closer to the front rotor gives a stronger vortex at hub blade with the separation that tends to increase from the hub area to the tip blade. Axial velocity contours in streamwise direction confirm the presence of a strong separation and vortex $\left(D_{1} / D_{2}=1.5\right)$. This phenomenon is further clarified in Fig. 14 and Fig. 16 which show the contours and resultant velocity vectors in the hub blade from the CRWT rotor CFD simulation results $\left(\mathrm{L} / \mathrm{D}_{1}=0.25\right)$ on the rotor diameter of $\mathrm{D}_{1} / \mathrm{D}_{2}=1.5$.

\section{Conclusion}

Analysis on aerodynamic performance and flow field phenomenon around the blade of counter-rotating wind turbine through rear rotor configurations by using various non-dimensional diameter and rotor distance in the range of TSR $=1$ to TSR $=10$ has been studied numerically (CFD simulation). Normalization of the power coefficient and the total power coefficient of CRWT on SRWT tends to decrease with the addition of the rotor diameter ratio. Whereas with the addition of the rotor axial distance, the front rotor CRWT performance continues to increase, while the rear rotor tends to decrease. The increase and decrease of the second rotor power coefficient of the CRWT provide an increase in combined performance or CRWT to SRWT with increasing the axial distance of the rotor. The normalized power coefficient of the front rotor, rear rotor, and combined rotor (CRWT) to the single rotor showed a strong influence of the rear rotor configuration with the addition of TSR. A larger average normalized power coefficient takes place at a rotor diameter ratio or $\mathrm{D}_{1} / \mathrm{D}_{2}=1.0$ with $\mathrm{L} / \mathrm{D}_{1}=0.75$ by 1.221 . It is about $22.1 \%$ increased to the SRWT for the given TSR range. However, for normalized total power coefficient or $\mathrm{C}_{\mathrm{P} \text {-tot }}$ occur at a diameter ratio of $\mathrm{D}_{1} / \mathrm{D}_{2}=0.5$ by 2.196 for rotor distance ratio or $\mathrm{L} / \mathrm{D}_{1}$ of 0.5 .

At a fairly close rotor distance $\left(\mathrm{L} / \mathrm{D}_{1}=0.25\right)$, the presence of high flow velocity by the rear rotor causes a low rate of recovery of wind speed so that a high decrease in speed. Whereas, if the distance of the rotors is increased, the rate of recovery of flow velocity becomes better and the interference velocity of flow by the rear rotor decreases. Axial velocity contours around CRWT with a diameter ratio of $\mathrm{D}_{1} / \mathrm{D}_{2}>0.5$ and a closer rotor distance provide a stronger vortex at the hub with a tendency to increase from the hub area to the tip of the blade. Axial velocity contours and vortices in streamwise direction confirm the presence of strong separation and vortices $\left(D_{1} / D_{2}=1.5\right)$. The higher the TSR, the movement of the vortex tip moves closer to the tip blade which has the effect of increasing the loss of flow in the area.

\section{Nomenclature}

A $=$ rotor cross-sectional area $\left(\mathrm{m}^{2}\right)$ 


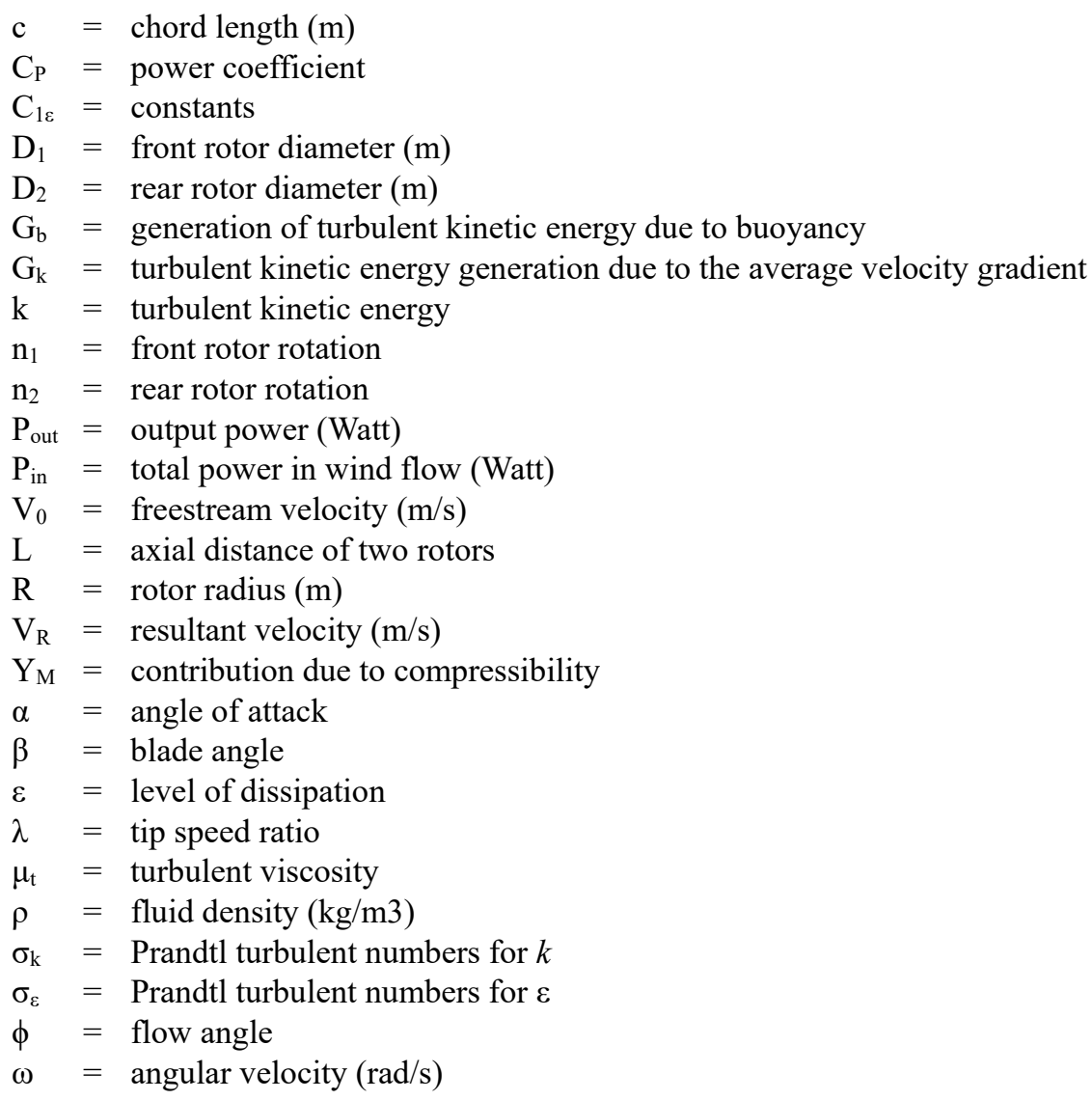

\section{Acknowledgments}

The authors thank the Ministry of Research, Tech., and Higher Education of the Republic of Indonesia for supporting this research and paper through The Grant 2018.

\section{References}

Appa, K. (2002). Counter rotating wind turbine system, Energy Innovations Small Grant (EISG) Program Technical Report. Sacramento, California, US.

Bartl, J., \& Sætran, L. (2017). Blind test comparison of the performance and wake flow between two in-line wind turbines exposed to different turbulent inflow conditions. Wind Energy Science, 2(1), 55-76. https://doi.org/10.5194/wes-2-55-2017

Burmester, S., Gueydon, S., \& Make, M. (2016). Determination of Scaled Wind Turbine Rotor Characteristics from Three Dimensional RANS Calculations. In Journal of Physics: Conference Series (Vol. 753, p. 82003). IOP Publishing. https://doi.org/10.1088/1742-6596/753/8/082003

Fluent Inc. (2006). Modeling Turbulent Flows. ANSYS.Inc., 6-2, 6-49.

Habash, R. W. Y., Groza, V., Yang, Y., Blouin, C., \& Guillemette, P. (2011). Performance of a contrarotating small wind energy converter. ISRN Mechanical Engineering, 2011. https://doi.org/10.5402/2011/828739

Hallanger, A., \& Sand, I. O. (2013). CFD wake modelling with a BEM wind turbine sub-model. Modeling, Identification and Control, 34(1), 19-33. https://doi.org/10.4173/mic.2013.1.3

Hoang, A. D., \& Yang, C. J. (2013). An evaluation of the performance of $10 \mathrm{kw}$ counter-rotating wind turbine using CFD simulation. EWEA 2013.

Hu, H., Wei, T., \& Wang, Z. (2016). An Experimental Study on the Wake Characteristics of Dual-Rotor Wind Turbines by Using a Stereoscopic PIV Technique. In 34th AIAA Applied Aerodynamics Conference (p. 3128). https://doi.org/10.2514/6.2016-3128

Hu, H., Yuan, W., Ozbay, A., \& Tian, W. (2013). An experimental investigation on the effects of turbine rotation directions on the wake interference of wind turbines. In 51st AIAA Aerospace Sciences Meeting including the New Horizons Forum and Aerospace Exposition (p. 607). https://doi.org/10.2514/6.2013-607 
Hwang, B. H., Lee, S. M., \& Soogab. (2013). Optimization of Counter Rotating Wind Turbine using Blade Element \& Momentum Theory. Journal of Renewable and Sustainable Energy, 5(5). https://doi.org/10.1063/1.4826940

Jung, S. N., No, T. S., \& Ryu, K. W. (2005). Aerodynamic performance prediction of a $30 \mathrm{~kW}$ counter-rotating wind turbine system. Renewable Energy, 30(5), 631-644. https://doi.org/10.1016/j.renene.2004.07.005

Kanemoto, T., \& Galal, A. M. (2006). Development of intelligent wind turbine generator with tandem wind rotors and double rotational armatures. JSME International Journal Series B Fluids and Thermal Engineering, 49(2), 450-457. https://doi.org/10.1299/jsmeb.49.450

Koehuan, V. A., Sugiyono, \& Kamal, S. (2017). Investigation of Counter-Rotating Wind Turbine Performance using Computational Fluid Dynamics Simulation. In IOP Conference Series: Materials Science and Engineering (Vol. 267, p. 12034). IOP Publishing. https://doi.org/10.1088/1757-899X/267/1/012034

Koehuan, V. A., Sugiyono, \& Kamal, S. (2017). The Near Wake Interference Effect on the Performance of Counterrotating Wind Turbine (CRWT). In The 9th International Conference on Thermofluids 2017 (Vol. 9). Yogyakarta. https://doi.org/10.1063/1.5049991

Krogstad, P. Å., \& Eriksen, P. E. (2013). "Blind test" calculations of the performance and wake development for a model wind turbine. Renewable Energy, 50, 325-333. https://doi.org/10.1016/j.renene.2012.06.044

Kumar, P. S., Abraham, A., Bensingh, R. J., \& Ilangovan, S. (2013). Computational and experimental analysis of a counter-rotating wind turbine system. Journal of Scientific and Industrial Research (JSIR), 72(05), 300306. https://doi.org/http://hdl.handle.net/123456789/17441

Kumar, P. S., Bensingh, R. J., \& Abraham, A. (2012). Computational analysis of $30 \mathrm{Kw}$ contra rotor wind turbine. ISRN Renewable Energy, 2012, p.5. https://doi.org/10.5402/2012/939878

Lee, S., Kim, H., \& Lee, S. (2010). Analysis of aerodynamic characteristics on a counter-rotating wind turbine. Current Applied Physics, 10(2), S339-S342. https://doi.org/10.1016/j.cap.2009.11.073

Lee, S., Kim, H., Son, E., \& Lee, S. (2012). Effects of design parameters on aerodynamic performance of a counterrotating wind turbine. Renewable Energy, 42, 140-144. https://doi.org/10.1016/j.renene.2011.08.046

Lee, S., Son, E., \& Lee, S. (2013). Velocity interference in the rear rotor of a counter-rotating wind turbine. Renewable Energy, 54, 235-240. https://doi.org/10.1016/j.renene.2012.08.003

Make, M., \& Vaz, G. (2015). Analyzing scaling effects on offshore wind turbines using CFD. Renewable Energy, 83, 1326-1340. https://doi.org/10.1016/j.renene.2015.05.048

Massouh, F., \& Dobrev, I. (2014). Investigation of wind turbine flow and wake. Journal of Fluid Science and Technology, 9(3), JFST0025-JFST0025. https://doi.org/10.1299/jfst.2014jfst0025

Merchant, S., Gregg, J., Gravagne, I., \& Van Treuren, K. (2009). Wind Tunnel Analysis Of A Counter-Rotating Wind Turbine. In Proceedings of the 2009 ASEE Gulf-Southwest Annual Conference Baylor University, Copyright $($ 2009, American Society for Engineering Education.

Mitulet, L.-A., Oprina, G., Chihaia, R.-A., Nicolaie, S., Nedelcu, A., \& Popescu, M. (2015). Wind tunnel testing for a new experimental model of counter-rotating wind turbine. Procedia Engineering, 100, 1141-1149. https://doi.org/10.1016/j.proeng.2015.01.477

Mo, J. O., \& Lee, Y. H. (2012). CFD Investigation on the aerodynamic characteristics of a small-sized wind turbine of NREL PHASE VI operating with a stall-regulated method. Journal of Mechanical Science and Technology, 26(1), 81-92. https://doi.org/10.1007/s12206-011-1014-7

Moghadassian, B., Rosenberg, A., \& Sharma, A. (2016). Numerical investigation of aerodynamic performance and loads of a novel dual rotor wind turbine. Energies, 9(7), 571. https://doi.org/10.3390/en9070571

Ozbay, A., Tian, W., \& Hu, H. (2014a). A Comparative Study of the Wake Characteristics behind a Single-Rotor Wind Turbine and Dual-Rotor Wind Turbines. In 32nd AIAA Applied Aerodynamics Conference (p. 2282). https://doi.org/10.2514/6.2014-2282

Ozbay, A., Tian, W., \& Hu, H. (2014b). An experimental investigation on the aeromechanics and near wake characteristics of dual-rotor wind turbines (DRWTs). In 32nd ASME Wind Energy Symposium (p. 1085). https://doi.org/10.2514/6.2014-1085

Ozbay, A., Tian, W., \& Hu, H. (2016). Experimental investigation on the wake characteristics and aeromechanics of dual-rotor wind turbines. Journal of Engineering for Gas Turbines and Power, 138(4), 42602. 
https://doi.org/10.1115/1.4031476

Rosenberg, A., Selvaraj, S., \& Sharma, A. (2014). A novel dual-rotor turbine for increased wind energy capture. In Journal of Physics: Conference Series (Vol. 524, p. 12078). IOP Publishing. https://doi.org/10.1088/17426596/524/1/012078

Shen, W. Z., Zakkam, V. A. K., Sørensen, J. N., \& Appa, K. (2007). Analysis of counter-rotating wind turbines. In Journal of Physics: Conference Series (Vol. 75, p. 12003). IOP Publishing. https://doi.org/10.1088/1742$6596 / 75 / 1 / 012003$

Sorensen, J. N., \& Shen, W. Z. (2002). Numerical modeling of wind turbine wakes. Journal of Fluids Engineering, 124(2), 393-399. https://doi.org/10.1115/1.1471361

Taha, Z., Sugioyno, \& Sawada, T. (2010). A comparison of computational and experimental results of Wells turbine performance for wave energy conversion. Applied Ocean Research, 32(1), 83-90. https://doi.org/10.1016/j.apor.2010.04.002

Tran, T. T., Kim, D. H., \& Nguyen, B. H. (2015). Aerodynamic interference effect of huge wind turbine blades with periodic surge motions using overset grid-based computational fluid dynamics approach. Journal of Solar Energy Engineering, 137(6), 61003. https://doi.org/10.1115/1.4031184

Ushiyama, I., Shimota, T., Miura, Y., \& 1996. (1996). An experimental study of the two-staged wind turbines. Elsevier, 9(1-4), 909-912. https://doi.org/10.1016/0960-1481(96)88427-8

Usui, Y., Kubo, K., \& Kanemoto, T. (2012). Intelligent wind power unit with tandem wind rotors and armatures (Optimization of front blade profile). Journal of Energy and Power Engineering, 6(11), 1791. https://doi.org/10.17265/1934-8975/2012.11.010

Vasel-Be-Hagh, A., \& Archer, C. L. (2017). Wind farms with counter-rotating wind turbines. Sustainable Energy Technologies and Assessments, 24, 19-30. https://doi.org/10.1016/j.seta.2016.10.004

Yuan, W., Tian, W., Ozbay, A., \& Hu, H. (2014). An experimental study on the effects of relative rotation direction on the wake interferences among tandem wind turbines. Science China Physics, Mechanics \& Astronomy, 57(5), 935-949. https://doi.org/10.1007/s11433-014-5429-x

Zhang, W., Markfort, C. D., \& Porté-Agel, F. (2012). Near-wake flow structure downwind of a wind turbine in a turbulent boundary layer. Experiments in Fluids, 52(5), 1219-1235. https://doi.org/10.1007/s00348-011$1250-8$

\section{Copyrights}

Copyright for this article is retained by the author(s), with first publication rights granted to the journal.

This is an open-access article distributed under the terms and conditions of the Creative Commons Attribution license (http://creativecommons.org/licenses/by/4.0/). 